\title{
Effects of gravitational darkening on the determination of fundamental parameters in fast-rotating B-type stars ${ }^{\star}$
}

\author{
Y. Frémat ${ }^{1}$, J. Zorec ${ }^{2}$, A.-M. Hubert ${ }^{3}$, and M. Floquet ${ }^{3}$ \\ 1 Royal Observatory of Belgium, 3 avenue circulaire, 1180 Bruxelles, Belgium \\ e-mail: yves.fremat@oma.be \\ 2 Institut d'Astrophysique de Paris, CNRS, 98bis Boulevard Arago, 75014 Paris, France \\ 3 Observatoire de Paris, Section d'Astrophysique de Meudon, GEPI, FRE K 2459, 5 place Jules Janssen, \\ 92195 Meudon Cedex, France
}

Received 22 October 2004 / Accepted 19 March 2005

\begin{abstract}
In this paper we develop a calculation code to account for the effects carried by fast rotation on the observed spectra of early-type stars. Stars are assumed to be in rigid rotation, and the grid of plane-parallel model atmospheres used to represent the gravitational darkening are calculated by means of a non-LTE approach. Attention is paid to the relation between the apparent and parent non-rotating counterpart stellar fundamental parameters and apparent, and true $V \sin i$ parameters as a function of the rotation rate $\Omega / \Omega_{\mathrm{c}}$, stellar mass, and inclination angle. It is shown that omitting of gravitational darkening in the analysis of chemical abundances of CNO elements can produce systematic overestimation or underestimation, depending on the lines used, rotational rate, and inclination angle. The proximity of Be stars to the critical rotation is revised while correcting not only the $V \sin i$ of $130 \mathrm{Be}$ stars, but also their effective temperature and gravity to account for stellar rotationally induced geometrical distortion and for the concomitant gravitational darkening effect. We concluded that the $V \sin i$ increase is accompanied by an even higher value for the stellar equatorial critical velocity, so that the most probable average rate of the angular velocity of Be stars attains $\Omega / \Omega_{\mathrm{c}} \simeq 0.88$.
\end{abstract}

Key words. stars: abundances - stars: atmospheres - stars: early-type - stars: emission-line, Be - stars: fundamental parameters

\section{Introduction}

Fast rotation produces polar flattening and equatorial stretching of stars, which in turn induce non-uniform surface gravity and temperature distributions: the gravity darkening effect (GD, Zeipel 1924). Owing to the levitation effect introduced by this rotation, rotating stars evolve as if they had a lower mass: their core density is higher, the total bolometric luminosity produced is lower and they spend a longer time on the main sequence evolutionary phase than slowly rotating stars with the same mass (Sackmann 1970; Bodenheimer 1971; Clement 1979; Moss \& Smith 1982; Meynet \& Maeder 2000). The geometrical deformation of the star and the GD further influence the apparent status of the star, which also depends on whether it has a radiative or convective envelope (Lucy 1967; Tuominen 1972; Smith \& Worley 1974; Claret 1998, 2000). As predicted by models of stellar evolution (Endal \& Sofia 1979; Pinsonneault et al. 1991; Heger \& Langer 2000; Meynet $\&$ Maeder 2000), fast rotation is further expected to generate several instabilities that contribute to redistributing the internal angular momentum. They thereby induce turbulent diffusion in

$\star$ Table 9 is only available in electronic form at http://www.edpsciences.org the stellar interior (Zahn 1992), which in massive objects drives the $\mathrm{CNO}$-cycled material from the core to the envelope and changes the atmospheric chemical composition in these stars (Meynet \& Maeder 2000).

\subsection{Rotation dependent spectra of early type stars}

Radiative and hydrostatic equilibrium conditions in atmospheres of rotating early-type stars require on each point on the stellar surface that the emitted bolometric flux be proportional to the local gravity $F \propto g_{\text {eff }}^{\beta}$ with $\beta \simeq 1$ if $T_{\text {eff }} \gtrsim 7000 \mathrm{~K}$ and $g_{\text {eff }}=g_{\text {grav }}-g_{\text {rot }}+g_{\text {rad }}$ (Zeipel 1924; Claret 2000). Although a non-local theory of radiative transfer in rotating stars does not reproduce von Zeipel's relation, meridional circulation and turbulence in surface layers recover the validity of this law (Smith $\&$ Worley 1974). For B-type star effective temperatures, the radiation pressure $g_{\text {rad }}$ can be neglected. Energy distributions, photometric indices, and spectral lines produced in gravitydarkened early type rotating stars were calculated by several authors, either for rigid (Collins 1963, 1965, 1966, 1968a,b, 1974; Hardorp \& Strittmatter 1968b, 1972, 1968a; Hardorp \& Scholz 1971; Collins \& Sonneborn 1977; Collins et al. 1991; Maeder \& Peytremann 1970, 1972) or for differential rotators 
with conservative internal rotational laws (Collins \& Smith 1985; Zorec 1986). A0 to F5-type rigid rotators were studied by Pérez Hernández et al. (1999). The effects of the GD on the $V \sin i$ determinations were explored by Stoeckley (1968) for rigid rotators and Zorec et al. (1988) for differential rotators. Stoeckley (1968) concluded that the reduced contribution to He I 4471 line broadening in the equatorial region may lead to underestimated rotational parameters in early-type stars, while Zorec et al. (1988a) obtained double-valued $V \sin i$ in star models with polar hollows. Recently, Townsend et al. (2004) discussed the GD effect on the $V \sin i$ values of rigid early-type rotators and concluded that classic $V \sin i$ determinations for B0 to B9-type stars can be underestimated by 12 to $33 \%$ at $\omega=\Omega / \Omega_{\mathrm{c}}=0.95$ rotational rates, if the He I 4471 line is used and from 9 to $17 \%$, if the $\mathrm{Mg}$ II 4481 line is studied.

\subsection{Aim of the present work}

Apart from the obvious rotation dependent-Doppler effect, four factors, at least, still concur to produce the observed line broadening in gravity-darkened fast rotators: a) changes in the surface non-uniform temperature of the continuous spectrum specific intensity; b) dependence of the intrinsic lines equivalent width on the local temperature and gravity; c) stretching of the isotemperature and isogravity regions, which implies that in strips of constant radial velocity for rather large Doppler displacements there can be temperatures close to that of the undistorted star; d) for high enough inclinations, the flattened stellar disc produces shortened constant Doppler displacement strips that tend to make the rotationally broadened line profile shallower. A look at the dependence of He I 4471 and Mg II 4481 line equivalent width on temperature and on gravity can be obtained in Didelon (1982) and Sect. 4.3 below. These lines are currently used to determine the $V \sin i$ parameters of early-type stars (Slettebak et al. 1975). From classic model atmospheres we find that at the He I 4471 and Mg II 4481 line wavelengths the continuum specific intensity scales as $I_{\mathrm{c}} \sim$ $T_{\text {eff }}^{a}$, with $a \sim 2.2$ if $T_{\text {eff }} \gtrsim 7500 \mathrm{~K}$. Since $T_{\text {eff }}(\mathrm{eq}) / T_{\text {eff }}($ pole $)=$ $\left(g_{\text {eq }} / g_{\text {pole }}\right)^{0.25}$, we infer that for $\omega=0.95$ the equatorial regions contribute less to the flux of the broadened line profile than in the pole by a factor $I_{\mathrm{c}}(\mathrm{eq}) / I_{\mathrm{c}}$ (pole) $\lesssim 0.2$. Line profiles are then expected to be narrower than those calculated with models where GD is neglected. At high aspect angles, effect d) compensates partially for this deepening. Depending on the temperature of the star, in fast rotators the change of the local $T_{\text {eff }}$ and $\log g$ with latitude produces the equivalent width of the He I 4471 line whether $W_{\mathrm{He} \mathrm{I}}($ eq $)>W_{\mathrm{He} \mathrm{I}}($ pole) or $W_{\mathrm{He} \mathrm{I}}(\mathrm{eq})<W_{\mathrm{He} \mathrm{I}}($ pole), while the equivalent width of the $\mathrm{Mg}$ II 4481 line is always $W_{\mathrm{Mg} \text { II }}(\mathrm{eq})>W_{\mathrm{Mg} \text { II }}$ (pole). Note that for the He I 4471 line, we actually mean the blend He I 4471+He I 4470, where the second line is a forbidden component and has a somewhat different sensitivity to fundamental parameters than does the permitted $\lambda 4471$ component. Since the He I 4471 and $\mathrm{Mg}$ II 4481 lines also respond in a different way to the GD and because they overlap partially, the $V \sin i$ determination demands that we proceed to fit both lines simultaneously.
The aim of this paper is thus to take into account, as properly as possible, the changes of the stellar radii $R_{\text {pole }}$ and $R_{\text {eq }}$ and of the global bolometric luminosity with $\omega$ in the calculation of the emitted radiation fluxes. We will also fit both He I 4471 and Mg II 4481 lines, in order to determine up to what degree the $V \sin i$ parameters of early-type fast rotators are underestimated if they are obtained once assuming that rotational distortion and GD can be neglected. For Be stars it is of particular interest to know whether they are near-critical rotators (Townsend et al. 2004). In this paper, we will also discuss the effect of the rapid rigid rotation on determination of the stellar effective temperature, surface gravity, and chemical composition.

\section{Model atmospheres}

Fast rotation flattens the star and produces non-uniform density and temperature distributions on its surface. To take the first order effects of this flattening on the stellar spectrum of B-type stars into account, we adopted a method similar to the one described by Collins et al. (1991), but modified to introduce the changes explained in Sect. 2.1. In this approach, using a computer code hereafter named FASTROT, the stellar photosphere is replaced by a mesh of plane-parallel model atmospheres each depending on the local temperature and surface gravity (Sect. 2.2).

\subsection{Flattening and gravitational darkening}

We assume that the stars are rigid rotators without any surface latitudinal differential rotation component. We adopted the Roche approximation for the stellar surface equipotentials; i.e. in the gravity potential the dipole and higher order terms are neglected (Tassoul 1978). In the expression of surface effective gravity we also neglected the latitude-dependent radiation pressure term $\left(g_{\mathrm{rad}}=0\right.$; Maeder $\&$ Meynet 2000). From the Roche equipotentials it follows that the ratio between the equatorial to the polar radii at the rotational rate $\omega=\Omega / \Omega_{\mathrm{c}}\left(\Omega_{\mathrm{c}}=\right.$ critical angular velocity) is given by:

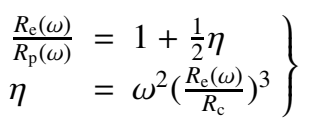

where $R_{\mathrm{c}}$ is the equatorial radius at critical rotation $(\omega=1)$.

In what follows we use the $\Omega / \Omega_{\mathrm{c}}$ to characterize the rotation rate instead of $V_{\mathrm{e}} / V_{\mathrm{c}}$, where $V_{\mathrm{e}}$ and $V_{\mathrm{c}}$ are the actual and critical linear equatorial rotation velocities, respectively. The main reason for this choice is that as we assume stars are rigid rotators, the angular velocity $\Omega$, which is the same over the whole star (surface and interior) and independent of any other stellar quantity, it can be considered as an independent fundamental parameter. We note, however, that the stellar rotational distortion and the associated gravity darkening effect are controlled by the parameter $\eta$ given in Eq. (1). Though $\Omega / \Omega_{\mathrm{c}}$, $V_{\mathrm{e}} / V_{\mathrm{c}}$, and $\eta$ are functions having the same germs 0 and 1 , it is $\Omega / \Omega_{\mathrm{c}}<V_{\mathrm{e}} / V_{\mathrm{c}}<\eta$ in the open interval $0<\Omega / \Omega_{\mathrm{c}}<1$.

In previous works it is often assumed that $R_{\mathrm{p}}(\omega)=R_{0}$, where $R_{0}$ is the radius of the star at rest (Collins et al. 1991; 
Reiners 2003; Townsend et al. 2004). In the present study we adopted instead the following interpolation expression for $R_{\mathrm{p}}$, derived from the calculations performed by Bodenheimer (1971), Clement (1979), and Zorec et al. (1988):

$$
\left.\begin{array}{rl}
\frac{R_{\mathrm{p}}(\omega)}{R_{\mathrm{o}}} & =1-P(M) \tau \\
P(M) & =5.66+\frac{9.43}{M^{2}}, \quad \text { for } M \gtrsim 2
\end{array}\right\}
$$

where $M$ is the stellar mass in solar units, $\tau=K /|W|$ with $K$ the rotational kinetic energy, and $W$ is the stellar gravitational potential energy. A rough relation between $\tau$ and $\eta$ is given by $\tau \simeq\left[0.0072+0.008 \eta^{1 / 2}\right] \eta^{1 / 2}$. Although Eq. (1) implies $R_{\mathrm{c}}=1.5 R_{0}$ at critical rotation, as from Eq. (2) $R_{\mathrm{p}}(\omega)<R_{0}$, depending on the B-type stellar mass, it is $R_{\mathrm{c}}=1.3 R_{0}$ to $1.4 R_{0}$, rather than $R_{\mathrm{c}}=1.5 R_{0}$, as usually assumed in the literature.

The total bolometric luminosity produced in the stellar core was assumed to be given by:

$L\left(\omega, M, g_{0}\right)=L_{0}\left(M, g_{0}\right) f_{\mathrm{L}}(\tau, M)$

where $L_{0}$ is the bolometric luminosity of an homologous nonrotating star with mass $M$ and rest surface gravity $g_{0}$. The expression for factor $f_{\mathrm{L}}$ was derived from Clement's (1979) models for $M \gtrsim 1.5$ :

$$
\left.\begin{array}{l}
f_{\mathrm{L}}=a(M)+[1-a(M)] \mathrm{e}^{-b(M) \tau} \\
a(M)=0.675+0.046 M^{1 / 2} \\
b(M)=52.71+20.63 M^{1 / 2}
\end{array}\right\} .
$$

The quoted models (Bodenheimer 1971; Clement 1979; Zorec et al. 1988b) were calculated for stars with internal conservative differential rotation laws and for energy ratios $\tau$ that reach the global secular stability limit $\tau=K /|W| \simeq 0.14$ and even higher. In all these models the core is supposed to rotate as a rigid body. Moreover, these calculations show that the radii ratio $R_{\mathrm{p}}(\omega) / R_{\mathrm{c}}$ and function $f_{\mathrm{L}}$ do not depend on the rotation law in the stellar envelope (Zorec 1986). Nevertheless, the interpolation relations (2) and (4) are for $0 \leq \tau=K /|W| \lesssim 0.03$, with a range of $\tau$ values which are suited for rigid rotators, including the rigid critical rotation, where $\tau=K /|W| \lesssim 0.02$ whatever the stellar mass in the $2 \lesssim M \lesssim 60$ range.

Relation (3) takes into account only the change in total bolometric luminosity due to the mechanic rotationally induced changes in the stellar core temperature and pressure (Sackmann 1970). Whenever possible, we checked that the changes introduced by mixing phenomena induced by the instabilities produced by the rotation (Endal \& Sofia 1976; Maeder \& Meynet 1996; Meynet \& Maeder 2000) do not introduce significant changes to the $f_{\mathrm{L}}$ given by Eq. (4).

In this work the GD law was written as:

$$
T_{\mathrm{eff}}^{4}(\theta, \omega)=\gamma\left(\omega, M, R_{0}\right) g^{\beta}(\theta, \omega)
$$

where $\theta$ is the colatitude angle; $M$ the stellar mass; $R_{0}$ the radius of the star at rest; $g$ the modulus of the gravity vector $\bar{g}=-\bar{\nabla} \Phi$ with $\Phi$ being the Roche potential; and $\beta$ was considered $=1$ for all local $T_{\text {eff }}(\theta, \omega) \gtrsim 7000 \mathrm{~K}$. For lower local $T_{\text {eff }}, \beta$ was interpolated in Claret (1998). The factor $\gamma$ was calculated for each $\omega$ as follows:

$\gamma=\frac{L\left(\omega, M, g_{0}\right)}{\sigma \int_{\mathcal{S}} g^{\beta} \mathrm{d} \mathcal{S}}$ where $\sigma$ is the Štefan-Boltzmann constant and $\mathcal{S}$ the total area of the surface Roche equipotential. From Eq. (3) it follows that the global effective temperature $\overline{T_{\mathrm{eff}}}=[L(\omega) / \sigma \mathcal{S}]^{1 / 4}$ is smaller than $T_{\text {eff }}^{0}=\left[L_{0} / 4 \pi \sigma R_{0}^{2}\right]^{1 / 4}$, the effective temperature of a non-rotating star with the same mass. On the other hand, relations (5) and (6) imply that $T_{\text {eff }}(\omega)_{\text {pole }}>\overline{T_{\text {eff }}}$. In the literature relation (5) is currently replaced by $T_{\text {eff }}^{4}(\theta, \omega)=T_{\text {eff }}^{4}(\theta=$ $0, \omega)[g(\theta, \omega) / g(\theta=0, \omega)]^{\beta}$, which hinders relating the apparent stellar fundamental parameters to those the star would have, if it were at rest. We note that the relations $T_{\text {eff }}(\theta=0, \omega)=T_{\text {eff }}^{0}$ and $g(\theta=0, \omega)=g_{0}$, where $T_{\mathrm{eff}}^{0}$ and $g_{0}$ are the fundamental parameters of the rotationless star, do not hold and that only the effective temperature and gravity averaged over the whole stellar hemisphere seen polar-on approach effective temperature $T_{\mathrm{eff}}^{0}$ and gravity $g_{0}$ roughly, i.e.: $\lim _{i \rightarrow 0}\left\langle T_{\mathrm{eff}}(\theta, \omega \mid i)\right\rangle \sim T_{\mathrm{eff}}^{0}$, and $\lim _{i \rightarrow 0}\langle g(\theta, \omega \mid i)\rangle \sim g_{0}$. In all cases it is $T_{\text {eff }}(\theta=0, \omega) \geq T_{\text {eff }}^{0}$ and $g(\theta=0, \omega) \geq g_{0}$.

\subsection{Local plane-parallel models}

The spectrum of a rotating star is represented as the intensity of radiation emitted per unit wavelength interval per steradian in the direction towards the observer defined by the aspect angle $i$ (inclination angle of the stellar rotation axis with respect to the line of sight). It is given by (Collins 1965; Maeder \& Peytremann 1970):

$L_{\lambda}(i, \omega)=2 \int_{0}^{\pi / 2} \mathrm{~d} \phi \int_{0}^{\pi} R^{2}(\theta) I_{\lambda}(\mu, \omega) \frac{|\mu|}{\cos \delta} \sin \theta \mathrm{d} \theta$

where $R(\theta)$ is the co-latitude $\theta$-dependent distance between the stellar center and the surface of the rotation ally-distorted star; $\mu=\mu(\phi, \omega)=\hat{n} \cdot \hat{B}$ ( $\hat{n}$ is the unit vector normal to the stellar surface and $\hat{B}$ is the unit vector representing the direction of the line of sight); $\cos \delta(\theta, \omega)=-\hat{n} . \hat{r}$ ( $\hat{r}$ is the unit vector in the direction of $R(\theta)) ; I_{\lambda}(\mu, \omega)$ is the $\mu$-dependent monochromatic specific radiation intensity calculated for the local effective temperatures $T_{\mathrm{eff}}(\theta, \omega)$ and surface gravities $g(\theta, \omega)$. The integration of Eq. (7) was performed using a Gauss-biquadrature of degree 96 in both angles $\theta$ and $\phi$, which is theoretically equivalent to a grid of 36481 surface elements of local plane-parallel model atmospheres. Each stellar surface point was characterized by its specific radial velocity in the observer's direction in order to take the corresponding Doppler shift in the spectral line into account.

The models we used to evaluate each local specific intensity $I_{\lambda}(\mu, \omega)$ were computed in two consecutive steps. To account in the most effective way for line-blanketing, the temperature structure of the atmospheres were computed as in Castelli \& Kurucz (2003) using the ATLAS9 computer code (Kurucz 1993). Non-LTE level populations were then computed for each of the atoms we considered using TLUSTY (Hubeny \& Lanz 1995) and keeping the temperature and density distributions obtained with ATLAS9 fixed.

Except for $\mathrm{C}$ II, the atomic models we used in this work were downloaded from TLUSTY's homepage (http:// tlusty.gsfc.nasa.gov) maintained by I. Hubeny and T. Lanz. Table 1 lists the ions that were introduced in our 
Table 1. Atomic models used.

\begin{tabular}{|c|c|c|}
\hline Atom & Ion & Description \\
\hline \multirow[t]{2}{*}{ Hydrogen } & $\mathrm{H}$ I & 8 levels +1 superlevel \\
\hline & $\mathrm{H}$ II & 1 level \\
\hline \multirow[t]{3}{*}{ Helium } & He I & 24 levels \\
\hline & He II & 20 levels \\
\hline & He III & 1 level \\
\hline \multirow[t]{4}{*}{ Carbon } & C II & 53 levels all individual levels \\
\hline & C III & 12 levels \\
\hline & C IV & 9 levels +4 superlevels \\
\hline & $\mathrm{C} \mathrm{V}$ & 1 level \\
\hline \multirow[t]{4}{*}{ Nitrogen } & $\mathrm{N}$ I & 13 levels \\
\hline & $\mathrm{N}$ II & 35 levels +14 superlevels \\
\hline & $\mathrm{N}$ III & 11 levels \\
\hline & $\mathrm{N}$ IV & 1 level \\
\hline \multirow[t]{4}{*}{ Oxygen } & O I & 14 levels +8 superlevels \\
\hline & O II & 36 levels + 12 superlevels \\
\hline & O III & 9 levels \\
\hline & O IV & 1 level \\
\hline \multirow{2}{*}{ Magnesium } & Mg II & 21 levels +4 superlevels \\
\hline & Mg III & 1 level \\
\hline
\end{tabular}

computations. C II was treated with the MODION IDL package developed by Varosi et al. (1995), and we adopted the atomic data (oscillator strengths, energy levels, and photoionization cross-sections) selected from the TOPBASE database (Cunto et al. 1993). It reproduces the results obtained by Sigut (1996).

In this way and for each spectral region studied in the present work, the specific intensity grids were computed for effective temperatures and surface gravities ranging from $15000 \mathrm{~K}$ to $27000 \mathrm{~K}$ and from 3.0 to 4.5 , respectively. For $T_{\mathrm{eff}}^{0}<15000 \mathrm{~K}$ and $T_{\mathrm{eff}}^{0}>27000 \mathrm{~K}\left(T_{\mathrm{eff}}^{0}\right.$ concern rotationless model atmospheres) we used LTE calculations and the OSTAR2002 model atmospheres grid (Lanz \& Hubeny 2003).

\section{Comparison of rotating with non-rotating models}

\subsection{Adopted procedure}

Hydrogen and helium line profiles, more particularly, the $\mathrm{H} \gamma$, $\lambda 4388 \mathrm{He} \mathrm{I}$, and $\lambda 4471 \mathrm{He}$ I lines, are often used to derive the effective temperature, surface gravity, and projected rotation velocity of B type stars. Among several reasons of their use, we have: 1) the line profiles and equivalent widths are very sensitive to effective temperature and surface gravity; 2) the blue helium lines are known to be somewhat less sensitive to LTE departures; 3 ) the broadening mechanisms of their line profiles have been well known for a long time (e.g.: Barnard et al. 1969; Leckrone 1971; Vidal et al. 1973; Mihalas et al. 1974).

Two grids of spectra ranging from $4250 \AA$ to $4500 \AA$ were computed. A first grid, GRIDA, was obtained using classical plane-parallel model atmospheres. A second grid, GRIDB, was calculated using FASTROT (Sect. 2) where stellar flattening and gravity darkening are taken into account. The sets of fundamental parameters considered in these grids are given in Table 2. $T_{\mathrm{eff}}^{0}$ and $g_{0}$ refer to the fundamental parameters of the
Table 2. Sets of parameters used in model grids.

\begin{tabular}{rcll}
\hline \hline GRIDA & & & \\
\hline $5000 \mathrm{~K} \leq$ & $T_{\text {eff }}^{0}$ & $\leq 40000 \mathrm{~K}$ & step $=1000 \mathrm{~K}$ \\
$2.5 \leq$ & $\log g_{0}$ & $\leq 5.0$ & step $=0.5$ \\
$0 \mathrm{~km} \mathrm{~s}^{-1} \leq$ & $V \sin i$ & $\leq 500 \mathrm{~km} \mathrm{~s}^{-1}$ & step $=50 \mathrm{~km} \mathrm{~s}^{-1}$ \\
\hline GRIDB & & & \\
\hline $12000 \mathrm{~K} \leq$ & $T_{\text {eff }}^{0}$ & $\leq 27000 \mathrm{~K}$ & step $=1000 \mathrm{~K}$ \\
$3.2 \leq$ & $\log g_{0}$ & $\leq 4.4$ & step $=0.2$ \\
$0^{0} \leq$ & $i$ & $\leq 90^{0}$ & step $=5^{\circ}$ \\
$0.50 \leq$ & $\Omega / \Omega_{\mathrm{c}}$ & $\leq 0.99$ & step $=0.05$ \\
\hline
\end{tabular}

Table 3. Spectral regions considered for the fits.

\begin{tabular}{cccc}
\hline \hline & \multicolumn{3}{c}{ CASE } \\
Spectral line & A & B & $\Delta \lambda(\AA)$ \\
\hline $\mathrm{H} \gamma$ & $\times$ & $\times$ & $4300-4375$ \\
$\lambda 4388 \mathrm{He}$ I & $\times$ & $\times$ & $4375-4400$ \\
$\lambda 4471 \mathrm{He}$ I & & $\times$ & $4460-4475$ \\
$\lambda 4471 \mathrm{He}$ I $+\lambda 4481 \mathrm{Mg}$ II & $\times$ & & $4460-4490$ \\
\hline
\end{tabular}

rotationless or parent non-rotating counterpart of the star (Sect. 2.1). Regarding the notation, it is worth noting that for GRIDA it holds $T_{\text {eff }}=T_{\text {eff }}^{0}$ and $\log g=\log g_{0}$.

In what follows we will determine the effective temperature, surface gravity and the projected rotational velocity of B-type stars using only the $\mathrm{H} \gamma$, HeI 4388, HeI 4471 and MgII 4481 lines. To this end we will use a fitting procedure based on a least-squares method that applies the MINUIT minimization package available at CERN. In order to know the confidence degree of this method, we fitted the spectra of the GRIDA reference library using synthetic spectra obtained from the same NLTE plane-parallel model atmospheres like the GRIDA spectra. The free or fit parameters used to this purpose were: the effective temperature, surface gravity, the projected rotational velocity, and the averaged flux ratio of the fitted to the reference spectrum. The $\chi^{2}$ deviation parameter was computed only for the selected spectral domains defined in Table 3 for CASE A. The comparison shown in Fig. 1 of parameters, obtained by the minimization procedure with the model GRIDA reference parameters, reveals that the agreement is quite satisfactory. However, this agreement is the best for the projected rotational velocity, whose accuracy is generally better than $4 \%$. The derived effective temperature approaches the reference values when $T_{\text {eff }} \sim 20000 \mathrm{~K}$ closest, but for other temperatures deviations never exceed $500 \mathrm{~K}$. The errors committed on $\log g$ are always lower than 0.05 dex, whatever the effective temperature.

To study the effects of fast rotation on determination of stellar fundamental parameters, we applied the same procedure as above to the GRIDB spectra, where the model parameters are $\left\{T_{\text {eff }}^{0}, \log g_{0}, i, \Omega / \Omega_{\mathrm{c}}\right\}$ or equivalently $\left\{T_{\text {eff }}^{0}, \log g_{0}, V \sin i\right.$, $\left.\Omega / \Omega_{\mathrm{c}}\right\}$. Since the fit of spectra produced by rotating stars can be done in two ways, we distinguish two sets of parameters. The reference model parameters proper to calculating FASTROT 

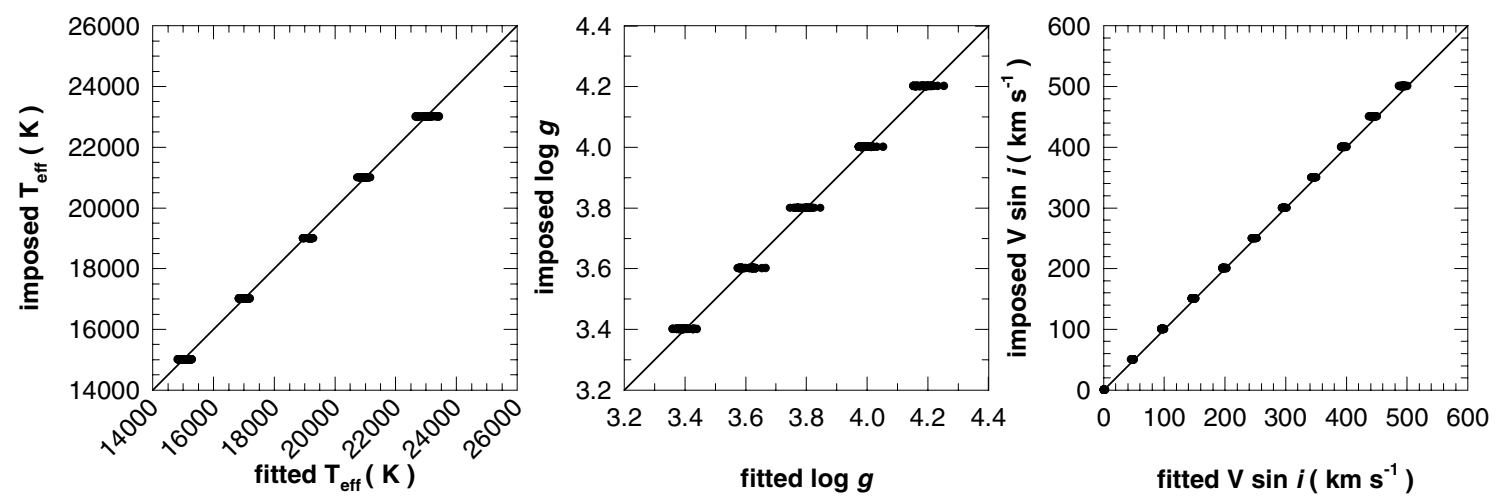

Fig. 1. Test of the adopted procedure: comparison of $T_{\text {eff }}, \log g$, and $V \sin i$ parameters obtained by the fitting procedure with the model parameters of GRIDA.

rotationally modified synthetic spectra, as well as those obtained by fitting the rotationally distorted spectra with them, are hereafter called parent non-rotating counterpart (pnrc) fundamental parameters and will be noted with a superscript "0", or a subscript "0" (i.e. $T_{\text {eff }}^{0}$ and $\log g_{0}$ ), while $V \sin i$ will represent the true projected rotation velocity of the star. Conversely, we call apparent fundamental parameters (e.g. $T_{\mathrm{eff}}^{\text {app. }}, \log g_{\text {app. }}$. and $V \sin i_{\text {app. }}$ ), those derived by fitting the GRIDB spectral lines with synthetic spectra issued from classical plane-parallel model atmospheres.

The fit of the $\mathrm{H} \gamma$, HeI 4388 and HeI 4471 lines was done either by including the MgII 4481 line or excluding it, in order to test the procedure's sensitivity to a change in the fitting criteria; this identifies CASE A or CASE B in Table 3, respectively.

\subsection{Reference parameters}

In the following sections, calculations will be done for several effective temperatures and surface gravities. To have a rough look at the spectral types that may be concerned as well as at the relations that exist between the pnrc fundamental parameters and the apparent ones - which are dependent on the stellar geometrical distortions and the concomitant GD - we list in Table 4 the reference parameters we used in the present work. This table also gives the polar effective temperature and the polar radius of the distorted stars for several values of the angular velocity ratio $\Omega / \Omega_{\mathrm{c}}$.

The MK spectral types adopted (Gray \& Corbally 1994) are for the effective temperatures and $\log g_{0}=4.0$ of the parent non-rotating stars. The stellar masses and radii for $\Omega / \Omega_{c}=0$ used to calculate the critical velocity $V_{\mathrm{c}}$ (cf. Sect. 2.1 ) were derived from stellar evolutionary tracks of non-rotating stars (Schaller et al. 1992).

\section{Apparent fundamental parameters}

Following the procedure described in Sect. 3.1, we obtained a grid of pnrc (i.e.: parameters belonging to the parent nonrotating stellar counter-part) and apparent fundamental parameters for different values of the angular velocity and inclination angle of the star. Since the aim of the present computations is to
Table 4. Polar temperature $\left(T_{\text {polar }}\right)$, polar radii $\left(R_{\mathrm{p}}\right)$, and critical velocities $\left(V_{\mathrm{c}}\right)$ as a function of $\Omega / \Omega_{\mathrm{c}}$ and for $\log g_{0}=4$.

\begin{tabular}{|c|c|c|c|c|c|c|}
\hline MK Type & $\begin{array}{c}T_{\text {eff }}^{0} \\
\mathrm{~K}\end{array}$ & $\begin{array}{c}\text { Mass } \\
M_{\odot}\end{array}$ & $\Omega / \Omega_{\mathrm{c}}$ & $\begin{array}{c}T_{\text {polar }} \\
\mathrm{K}\end{array}$ & $\begin{array}{l}R_{\mathrm{p}} \\
R_{\odot}\end{array}$ & $\begin{array}{c}V_{\mathrm{c}} \\
\mathrm{km} \mathrm{s}^{-1}\end{array}$ \\
\hline \multirow[t]{4}{*}{ B1 V } & 24620 & 10.53 & 0.00 & 24620 & 5.32 & 525 \\
\hline & & & 0.50 & 24932 & 5.22 & \\
\hline & & & 0.80 & 25887 & 5.10 & \\
\hline & & & 0.99 & 27593 & 4.88 & \\
\hline \multirow[t]{4}{*}{ B2 V } & 19500 & 6.87 & 0.00 & 19500 & 4.30 & 472 \\
\hline & & & 0.50 & 19736 & 4.21 & \\
\hline & & & 0.80 & 20471 & 4.11 & \\
\hline & & & 0.99 & 21773 & 3.94 & \\
\hline \multirow[t]{4}{*}{ B5 V } & 14000 & 4.15 & 0.00 & 14000 & 3.34 & 418 \\
\hline & & & 0.50 & 14176 & 3.27 & \\
\hline & & & 0.80 & 14708 & 3.19 & \\
\hline & & & 0.99 & 15609 & 3.04 & \\
\hline \multirow[t]{4}{*}{ B9 V } & 10720 & 2.86 & 0.00 & 10720 & 2.77 & 383 \\
\hline & & & 0.50 & 10870 & 2.71 & \\
\hline & & & 0.80 & 11300 & 2.63 & \\
\hline & & & 0.99 & 12007 & 2.50 & \\
\hline
\end{tabular}

provide a way to correct the observed fundamental parameters from gravitational darkening effects for a large parameterspace, we chose to present the effects due to fast rotation and the corresponding corrections as a function of $T_{\text {eff }}^{\text {app. }}, \log g_{\text {app. }}$, and $V \sin i_{\text {app. }}$ (e.g. Fig. 2), to make a direct link with the actually measured quantities, rather than against the pnrc fundamental parameters, which need to be determined from the corrections being sought. As the REFs are strongly dependent on the aspect angle $i$, we present them as a function of the observed $V \sin i_{\text {app. }}$.

\subsection{Effective temperature}

The typical effects of stellar flattening and GD on effective temperature are shown in Fig. 2 for different combinations of $T_{\text {eff }}^{0}$ and $\log g_{0}$, while the corrections $\Delta T_{\text {eff }}$ to add to the apparent effective temperature to get the pnrc effective temperature at different apparent $\Omega / \Omega_{\mathrm{c}}$, apparent $V \sin i$, and apparent $\log g$ regimes are plotted in Fig. 3 (upper panel). As expected, the $T_{\mathrm{eff}}^{\mathrm{app}}$ decreases with increasing $V \sin i_{\text {app. }}$. 


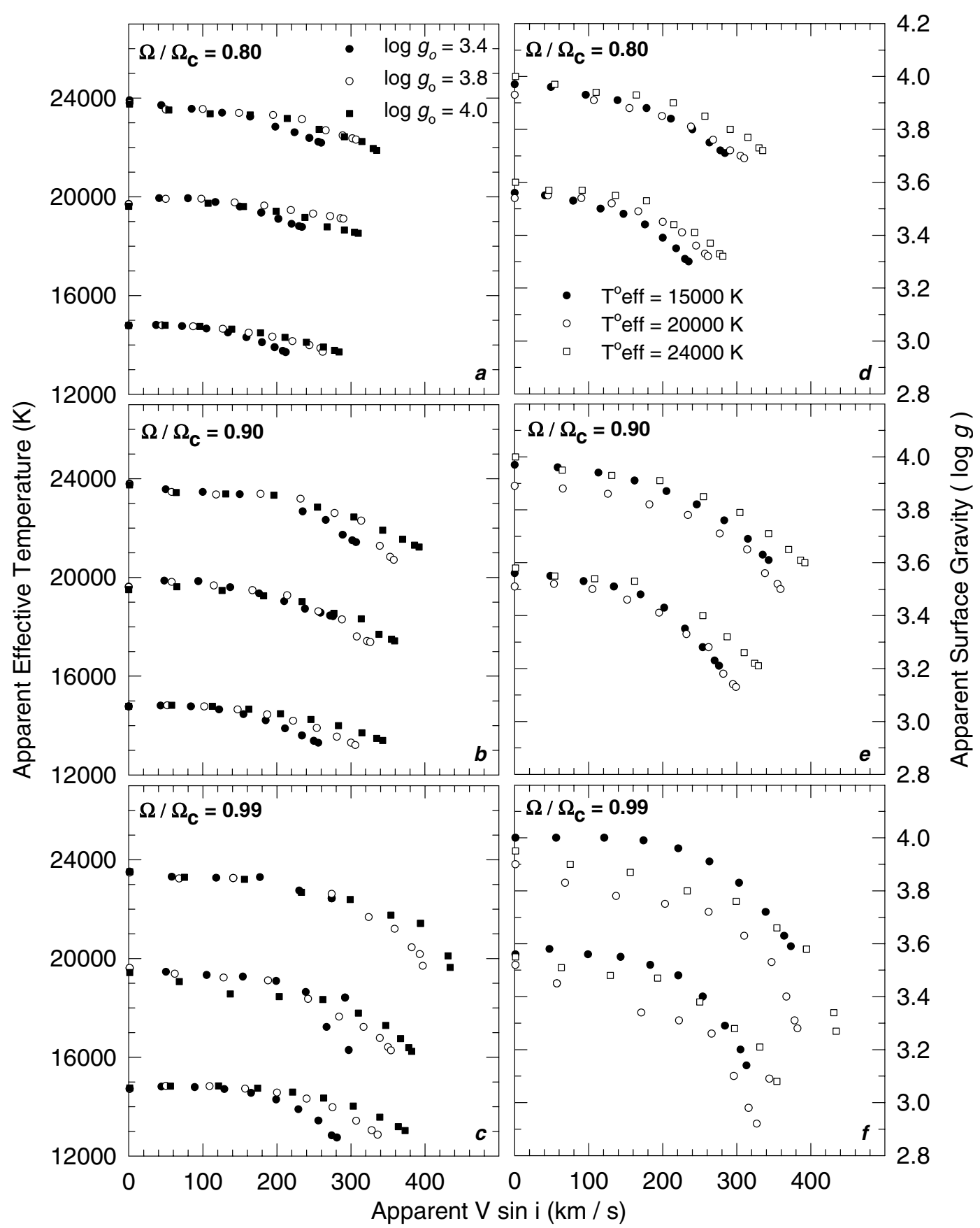

Fig. 2. Apparent effective temperature and surface gravity as a function of $V \sin i$ for different $p n r c\left(T_{\mathrm{eff}}^{0}, \log g_{0}\right)$ values and rotation rates $\Omega / \Omega_{\mathrm{c}}$.

Fast rotation therefore produces a lowering of the apparent effective temperature. Concerning the amplitude of this effect and its general behaviour, the following remarks can be made:

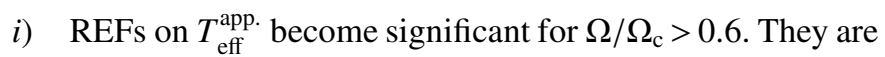
stronger and vary more rapidly with $V \sin i_{\text {app. }}$ the higher the values of $\Omega$.

ii) When $\Omega / \Omega_{\mathrm{c}}$ increases, REFs on $T_{\mathrm{eff}}^{\mathrm{app}}$ become more sensitive to surface gravity in the sense that they are larger when the gravity is lower.

iii) The relative lowering effect on the apparent $T_{\text {eff }}$ does not depend much on its pnrc value. In Table 5 this effect is given in percentages for different angular velocities in stars seen equator-on, where effects are stronger.

$i v$ ) When studying dwarf B-type stars, REFs on $T_{\mathrm{eff}}^{\text {app. }}$ can be ignored safely at $V \sin i_{\text {app. }}$ lower than $200 \mathrm{~km} \mathrm{~s}^{-1}$ and at $V \sin i_{\text {app. }} \lesssim 100 \mathrm{~km} \mathrm{~s}^{-1}$ for giant B-type stars.

\subsection{Surface gravity}

Corrections $\Delta \log g$ to add to the apparent surface gravity to get the pnrc surface gravity at different $\Omega / \Omega_{\mathrm{c}}$, apparent $V \sin i$ and apparent $T_{\text {eff }}$ regimes are plotted in Fig. 3 (lower panel). As shown in Fig. 2, stellar flattening and gravitational darkening 

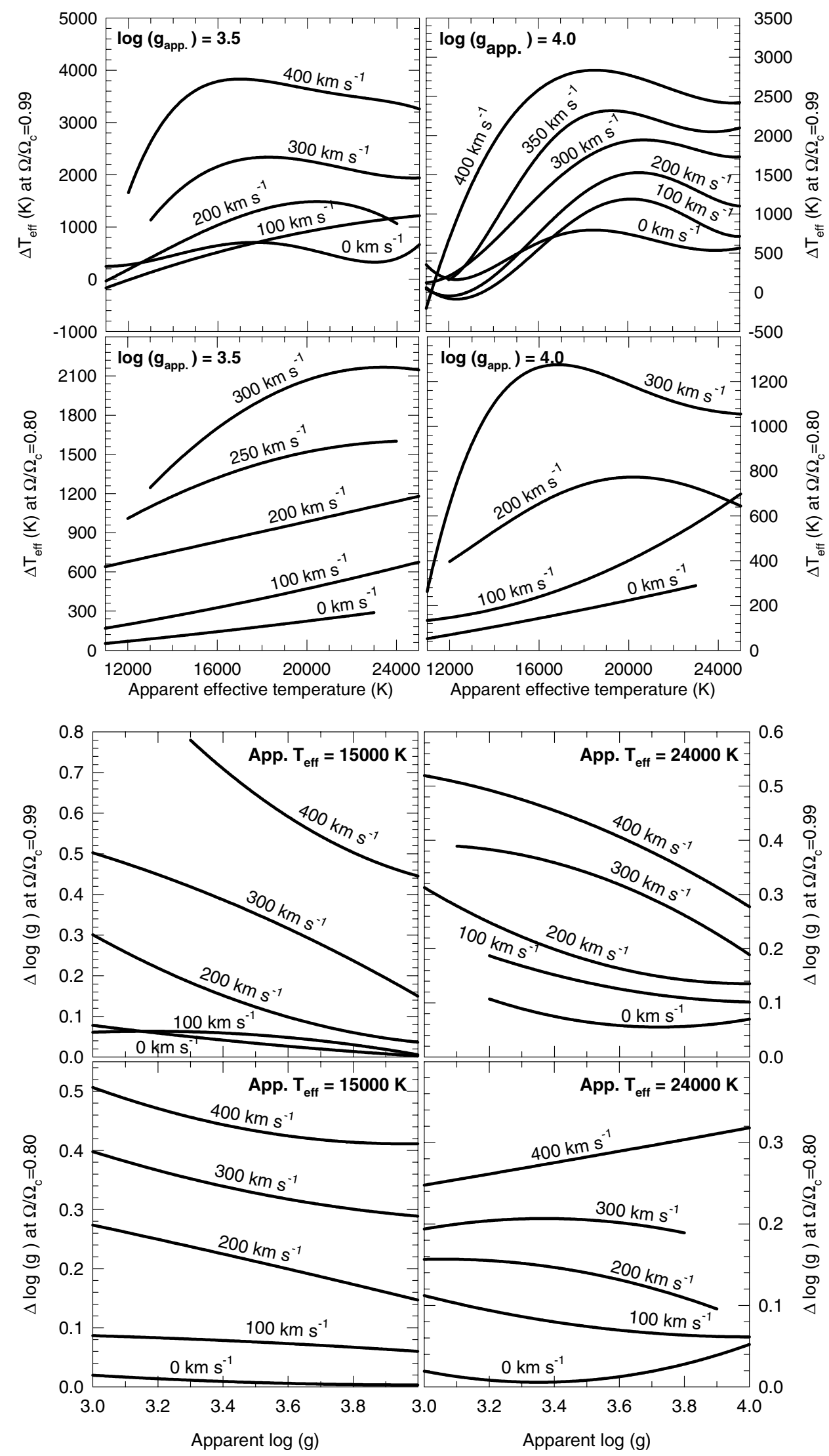

Fig. 3. Upper panel: corrections $\Delta T_{\text {eff }}$ to add to the apparent effective temperature $\left(T_{\text {eff }}^{\text {app. }}\right)$ for different $\Omega / \Omega_{\mathrm{c}}, V \sin i_{\text {app. }}$, and $\log g_{\text {app. }}$. Lower panel: corrections $\Delta \log g$ to add to the apparent surface gravity for different $\Omega / \Omega_{\mathrm{c}}$, apparent $V \sin i$, and apparent $T_{\text {eff }}$. 
Table 5. Effects of fast rotation in equator-on B type stars. The effective temperature $\left(R T_{\text {eff }}\right)$ and surface gravity $(R \log g)$ underestimation percentage are given against $\Omega / \Omega_{\mathrm{c}}$.

\begin{tabular}{crc}
\hline \hline$\Omega / \Omega_{\mathrm{c}}$ & $R T_{\text {eff }}$ & $R \log g$ \\
\hline 0.80 & $9 \%$ & $8 \%$ \\
0.90 & $12 \%$ & $10 \%$ \\
0.99 & $17 \%$ & $20 \%$ \\
\hline
\end{tabular}

lower the apparent surface gravity. The amplitude of this REF varies by obeying the following rules:

i) REFs on $\log g_{\text {app. }}$ become significant for $\Omega / \Omega_{\mathrm{c}}>0.6$. They are stronger and vary more rapidly with $V \sin i_{\text {app. }}$ at high $\Omega / \Omega_{\mathrm{c}}$ values.

ii) When $\Omega / \Omega_{\mathrm{c}} \lesssim 0.85$, REFs on $\log g_{\text {app. }}$ are weaker in stars with $T_{\mathrm{eff}}^{0} \gtrsim 20000 \mathrm{~K}$ than in those with $T_{\mathrm{eff}}^{0} \lesssim 20000 \mathrm{~K}$. In contrast, for $\Omega / \Omega_{\mathrm{c}} \gtrsim 0.85$, the apparent $\log g$ is more sensitive to REFs in stars with $T_{\text {eff }}^{0} \gtrsim 20000 \mathrm{~K}$ than in those with $T_{\text {eff }}^{0} \lesssim 20000 \mathrm{~K}$. This change in log $g$ sensitivity to REFs, according to $T_{\text {eff }}^{0}$ and $\Omega / \Omega_{\mathrm{c}}$, is due to hydrogen lines, which in the present approach are the main log $g$ indicators. In hot stars, the intensity of hydrogen lines is lowest, but it increases fast with the equatorial local $T_{\text {eff }}$ decrease produced by increasing values of $\Omega / \Omega_{\mathrm{c}}$.

iii) The lowering of $\log g_{\text {app. }}$ does not depend much on the pnrc $\log g$ value. It is given in percentages in Table 5 for different angular velocities in stars seen equator-on where effects are the stronger. In the more distorted cases, the change in $\log g_{\text {app. }}$ represents two luminosity classes.

$i v)$ In B-type stars with $T_{\text {eff }}^{\text {app. }} \lesssim 18000 \quad \mathrm{~K} \quad$ and $V \sin i_{\text {app. }} \lesssim 100 \mathrm{~km} \mathrm{~s}^{-1}$, REFs on log $g$ can be ignored.

\subsection{Projected rotation velocity}

The REFs on $V \sin i_{\text {app. }}$ become significant for $\Omega / \Omega_{\mathrm{c}} \gtrsim 0.70$. The spectral lines become progressively less broadened than expected from models of stars without GD, due to lower contribution to the line flux coming from the gravity-darkened equatorial regions towards the stellar limb. The magnitude of this effect depends on the sensitivity of the line studied to the local changes of $T_{\text {eff }}$ and $\log g$, as shown in Fig. 4 for the He I 4471 and for the $\mathrm{Mg}$ II 4481 lines.

At a given $\Omega / \Omega_{\mathrm{c}} \gtrsim 0.7$ and $T_{\text {eff }}^{0} \lessgtr 18000 \mathrm{~K}$, the broadening of the He I 4471 line tends to saturate for increasing values of $V \sin i_{\text {app. }}$. In stars cooler than $T_{\text {eff }}^{0} \simeq 18000 \mathrm{~K}$, the saturation of this broadening is also favored by enhanced contribution to the line flux from the polar regions, where the local $T_{\text {eff }}$ is increased by the GD. Figure 5 shows the apparent $V \sin i_{\mathrm{FFT}}$ against true $V \sin i$ for $\log g_{0}=4.0, \Omega / \Omega_{\mathrm{c}}=0.99$, and for different values of $T_{\text {eff }}^{0}$ when only the He I 4471 line (Fig. 5 a) is considered. The apparent $V \sin i$ parameter is obtained in this figure using the Fourier transform method applied to line profiles calculated with FASTROT. In the present work, the Fourier method is meant to represent the classical techniques of determining rotational parameters. We see that in a star with a $T_{\text {eff }}^{0}=14000 \mathrm{~K}$, a $V \sin i_{\text {app. }} \simeq 250 \mathrm{~km} \mathrm{~s}^{-1}$ value
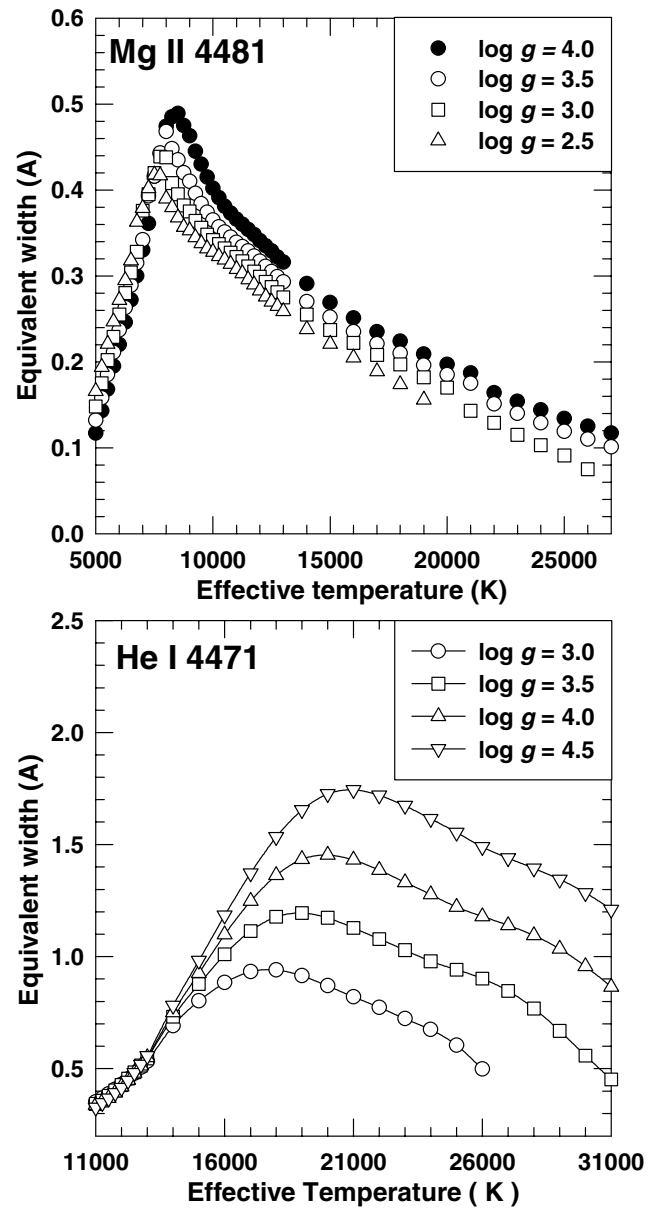

Fig. 4. Equivalent widths of the He I 4481 and Mg II 4481 spectral lines against effective temperature for different $\log g$ values. Computations were made assuming plane-parallel model atmospheres.

implies that the underestimation of the projected rotational velocity is $\delta V \sin i=(V \sin i)-\left(V \sin i_{\text {app. }}\right) \simeq 140 \mathrm{~km} \mathrm{~s}^{-1}$, if it rotates at $\Omega / \Omega_{\mathrm{c}}=0.99$. Underestimation $\delta V \sin i$ drops fast, as soon as $\Omega / \Omega_{\mathrm{c}}<0.99$, while the magnitude of the $V \sin i_{\text {app. }}$ saturation depends on the inclination angle, angular velocity, and also on the effective temperature (e.g. Figs. 5-8).

The same conclusions can be drawn for the Mg II 4481 line. However, in this case the above mentioned line broadening underestimation is less pronounced (Fig. 5b), due to the fact that the intensity of the $\mathrm{Mg}$ II 4481 line is smaller in the polar regions than in the equatorial, so that even the continuum intensity in the line wavelengths is stronger at the pole than at the equator. The differentiated response of the continuum and the intrinsic line intensity to $T_{\text {eff }}$ and $\log g$ compensate each other somewhat, producing thus a smaller $\delta V \sin i$.

The relation between the apparent and true $V \sin i$ derived from the Mg II 4481 is shown in Fig. 5, where we note in this figure that saturation effects are seen in the He I 4471 line for low $T_{\text {eff }}^{0}$ values and in the $\mathrm{Mg}$ II 4481 line when $T_{\text {eff }}^{0}$ is high. In the first case, the effect is due to a significant decrease in the equivalent width of the line for low effective temperatures (i.e. at the stellar equator). In the second case, the contrast of the continuum specific intensity between the pole and the 


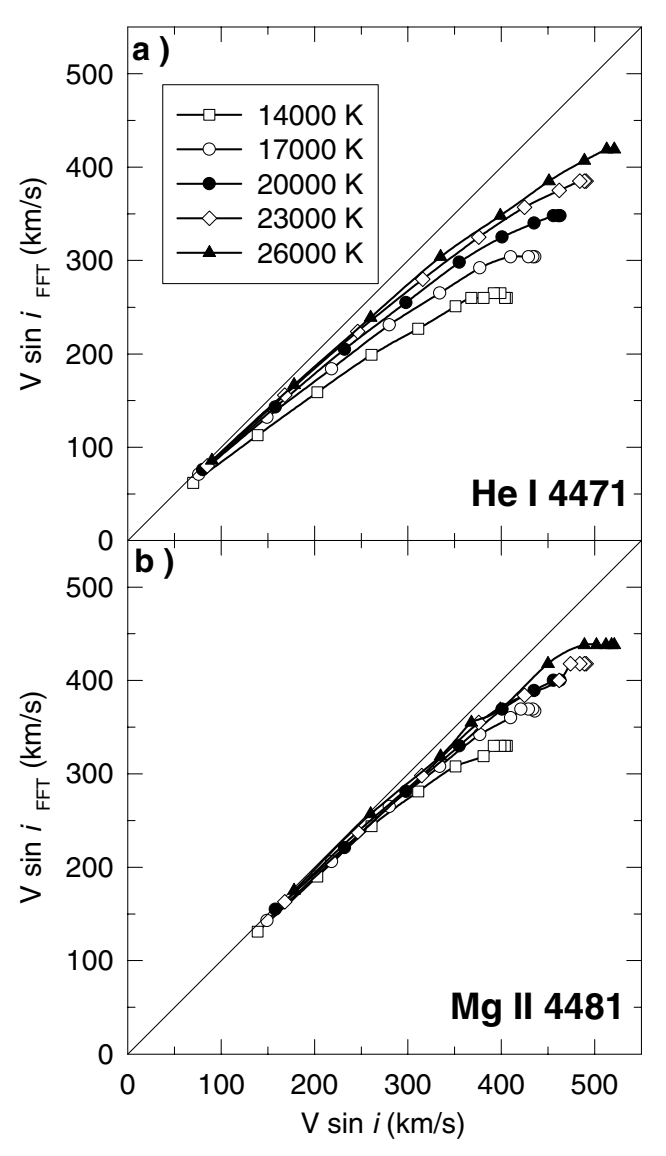

Fig. 5. Apparent $V \sin i$ derived using the Fourier Transform technique against the true $V \sin i$ considering individually the He I 4471 line (panel a)) and $\mathrm{Mg}$ II 4481 spectral line (panel b)). Computations are made assuming $\Omega / \Omega_{\mathrm{c}}=0.99$.

equator cannot be compensated for by the modest increase in the equivalent width of the line at lower equatorial temperatures (cf. Fig. 4).

As can be seen in Fig. 6, where $V \sin i_{\text {app. }}$ was derived following the procedure described in Sect. 3.1, the simultaneous fitting of various spectral lines, combined with the simultaneous determination of $T_{\text {eff }}^{\text {app. }}, \log g_{\text {app. }}$, and $V \sin i_{\text {app. }}$, somewhat limits the saturation effect and minimizes the underestimation of the projected rotation velocity at high inclinations and angular velocity rates. Figure 6 shows the relation between the apparent and true $V \sin i$ for $\Omega / \Omega_{\mathrm{c}}=0.80$, $\Omega / \Omega_{\mathrm{c}}=0.99, \log g_{0}=4.0$, and for different $T_{\mathrm{eff}}^{0}$ values when both He I 4471 and $\mathrm{Mg}$ II 4481 lines are taken into account for the $V \sin i$ determination. Considering the case $\Omega / \Omega_{\mathrm{c}}=0.99$ (i.e. $V_{\mathrm{e}} / V_{\mathrm{c}} \simeq 0.97$ ), we see that for whatever pnrc $T_{\text {eff }}$, the apparent $V \sin i$ underestimates the true rotation by $\delta V \sin i \lesssim 50 \mathrm{~km} \mathrm{~s}^{-1}$ as far as the apparent $V \sin i \lesssim 350 \mathrm{~km} \mathrm{~s}^{-1}$. Underestimations may then increase to $\delta V \sin i \simeq 80 \mathrm{~km} \mathrm{~s}^{-1}$ for apparent $V \sin i \gtrsim 350 \mathrm{~km} \mathrm{~s}^{-1}$. It is worth noting the peculiar behavior of this relation for $T_{\mathrm{eff}}^{0}=15000 \mathrm{~K}$. The enhanced contribution of the $\mathrm{Mg}$ II 4481 line to the blend He I 4471+Mg II 4481 at high inclinations means that the $V \sin i$ is most likely determined by the behavior of the Mg II 4481 which, as seen in Fig. 5, leads to a smaller $\delta V \sin i$ than the He I line alone. It is then crucial that for stars with $T_{\text {eff }}^{0} \lesssim 15000 \mathrm{~K}$, both He I 4471

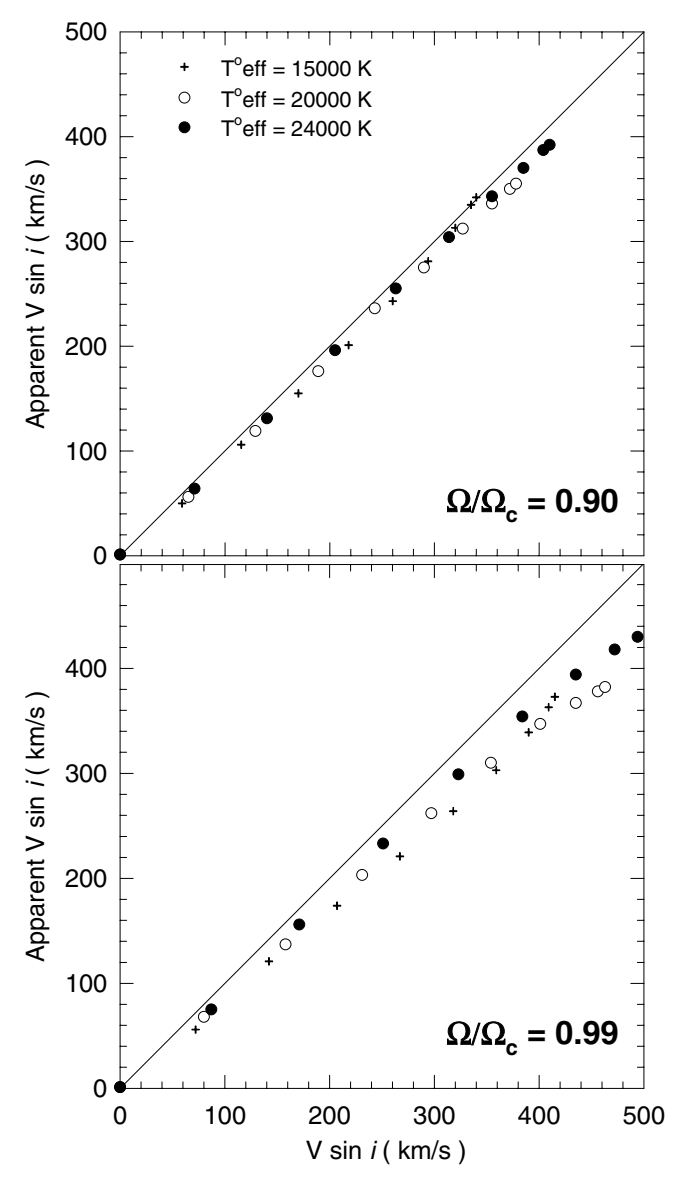

Fig. 6. Comparison between true and apparent $V \sin i$ in fast-rotating stars assuming $\log g_{\text {app. }}=4$. The fitted regions correspond to CASE A of Table 3.

and $\mathrm{Mg}$ II 4481 are considered together in the $V$ sin icalculation. On the contrary, for $T_{\mathrm{eff}}^{0} \gtrsim 20000 \mathrm{~K}$, the addition of $\lambda 4481$ $\mathrm{Mg}$ II in the fit does not significantly increase the accuracy of the $V \sin i$ measurements. Figure 6 also shows that for high enough $\Omega / \Omega_{\mathrm{c}}$ ratios the underestimation $\delta V \sin i$ is a function of $T_{\text {eff }}^{0}$.

As the use of the $V \sin i$ parameter at a given $\Omega / \Omega_{\mathrm{c}}$ hides the effect of the aspect angle on the measured $V \sin i$, we plotted the behaviour of the apparent $V \sin i$ in Fig. 7 for a pnrc B2 V spectral type against its true value at different fixed inclination angles $(\sin i)$. We note that the underestimation at higher $V \sin i$ is also very sensitive to the $\sin i$.

The incidence of $\log g_{0}$ on the underestimation $\delta V \sin i$ is further shown in Fig. 8. In this figure the relation between $\delta V \sin i$ against the true $V \sin i$ is given for different pnrc effective temperatures and surface gravities. It is seen that as long as $V \sin i \lesssim 300 \mathrm{~km} \mathrm{~s}^{-1}$, the $\delta V \sin i$ is almost independent of gravity. As soon as $V \sin i \gtrsim 300 \mathrm{~km} \mathrm{~s}^{-1}$, not only do the $\delta V \sin i$ differences depend strongly on $\log g_{0}$, but depending on the effective temperature, it shows two different behaviors. For temperatures $T_{\mathrm{eff}}^{0} \lesssim 20000 \mathrm{~K}, \delta V \sin i$ begins to decrease, while for $T_{\mathrm{eff}}^{0} \gtrsim 20000 \mathrm{~K}$ it increases even faster. In Fig. 8 the curve corresponding to $\log g_{0}=3.4$ is incorrectly determined for high 


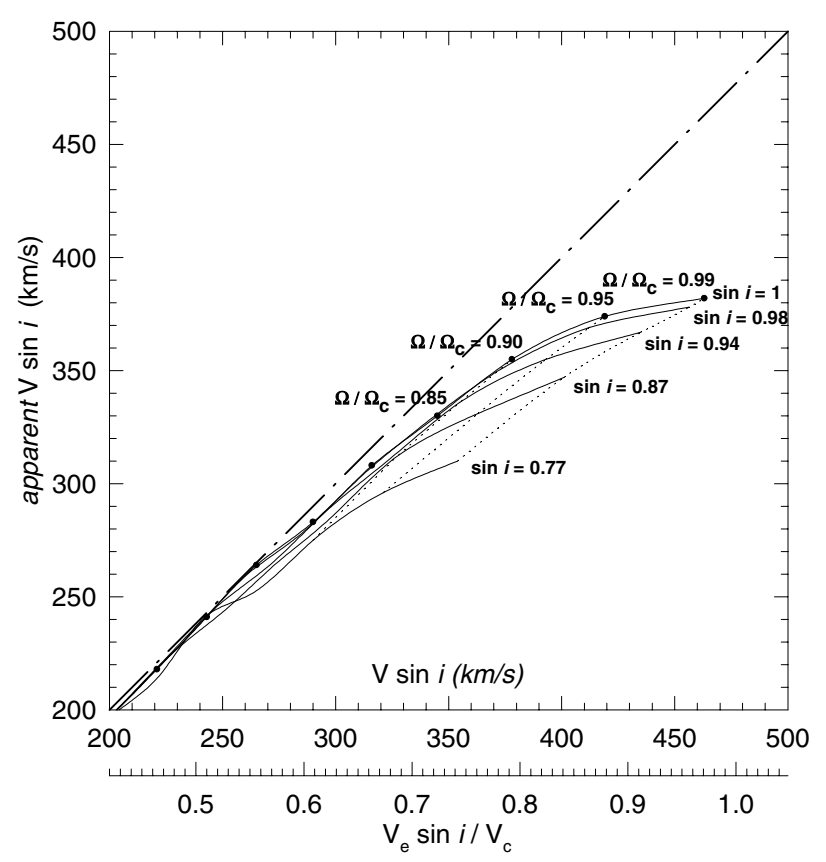

Fig. 7. Apparent and true $V \sin i$ values at $T_{\text {eff }}^{0}=20000 \mathrm{~K}, \log g_{0}=4.0$, and for different values of sin $i$ (full-line curves) and of $\Omega / \Omega_{\mathrm{c}}$ (dotted curves).

$V \sin i$ values, because the gravity decreases rapidly to very low values at the equator.

\section{Effects of fast rotation in other spectral regions}

It is very important to know whether plane-parallel model atmospheres calculated for the apparent fundamental parameters can describe the whole observed spectrum of a rotating star accurately enough. This matters not only for the study of the stellar chemical composition, but also for the energy distribution representation, which frequently enters into the fundamental parameter determination. This is important, in particular, for fast-rotating early type stars, whose evolution is faster than that of less massive stars, so that they are found in different evolutionary stages and can be used to test models of stellar evolution.

\subsection{Chemical composition}

In order to verify the reliability of using computed stellar spectra that ignore GD, we studied wavelength domains that contain several helium and hydrogen lines, as well as CNO spectral lines. We compared apparent spectra, i.e. those computed with a plane-parallel model atmosphere using the apparent fundamental parameters given in Table 7 with pnrc spectra (i.e. those calculated using FASTROT for $T_{\mathrm{eff}}^{0}=20000 \mathrm{~K}$, $\log g_{0}=4$ ) and several inclination angles $\Omega / \Omega_{\mathrm{c}}=0.99$. This comparison for hydrogen and helium lines is shown in Fig. 9. The same type of comparison for CNO elements is shown in Fig. 10. It appears then that in general REFs can be ignored for stars with $V \sin i \lesssim 150$, which correspond either to slow rotators or pole-on fast-rotators. However, in fast-rotating stars seen at high inclination angles, i.e.: large $V \sin i$ values, discrepancies

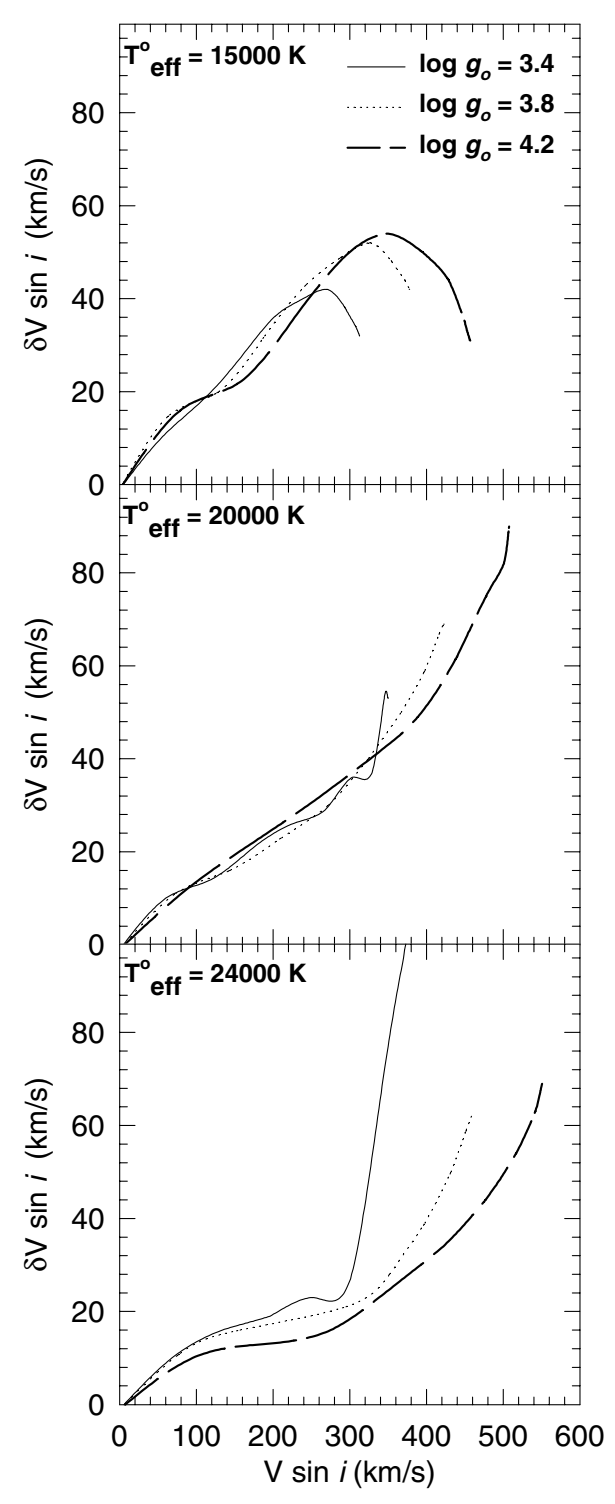

Fig. 8. $V \sin i$ lowering $(\delta V \sin i)$ as a function of the true $V \sin i$ for different true effective temperature and true $\log g$ values.

between both series of models can be significant. Thus, the chemical composition of objects seen at high inclinations, which are also the most frequently found, must be studied using models adapted for rotating stars.

Panel a) of Fig. 9 shows the helium and hydrogen lines used to determine the apparent $T_{\text {eff }}$ and $\log g$, as noted in Sect. 3.1. The spectral regions where the fit was actually performed are marked on top of spectra with thick horizontal lines. In general, these same plane-parallel models calculated assuming apparent fundamental parameters quite satisfactorily represent the helium and hydrogen lines in other spectral regions (see panel b) in Fig. 9).

The behaviour of the CNO spectral lines as function of the inclination angle and angular velocity is, however, more complex, as shown in Fig. 10. In Fig. 11, we report the overabundance of oxygen and nitrogen derived for models corresponding to an apparent B2 IV star $\left(T_{\mathrm{eff}}^{\mathrm{app}}=19000 \mathrm{~K}\right.$ and $\left.\log g_{\text {app. }}=3.5\right)$ but for different values of $\Omega / \Omega_{\mathrm{c}}$ and two 

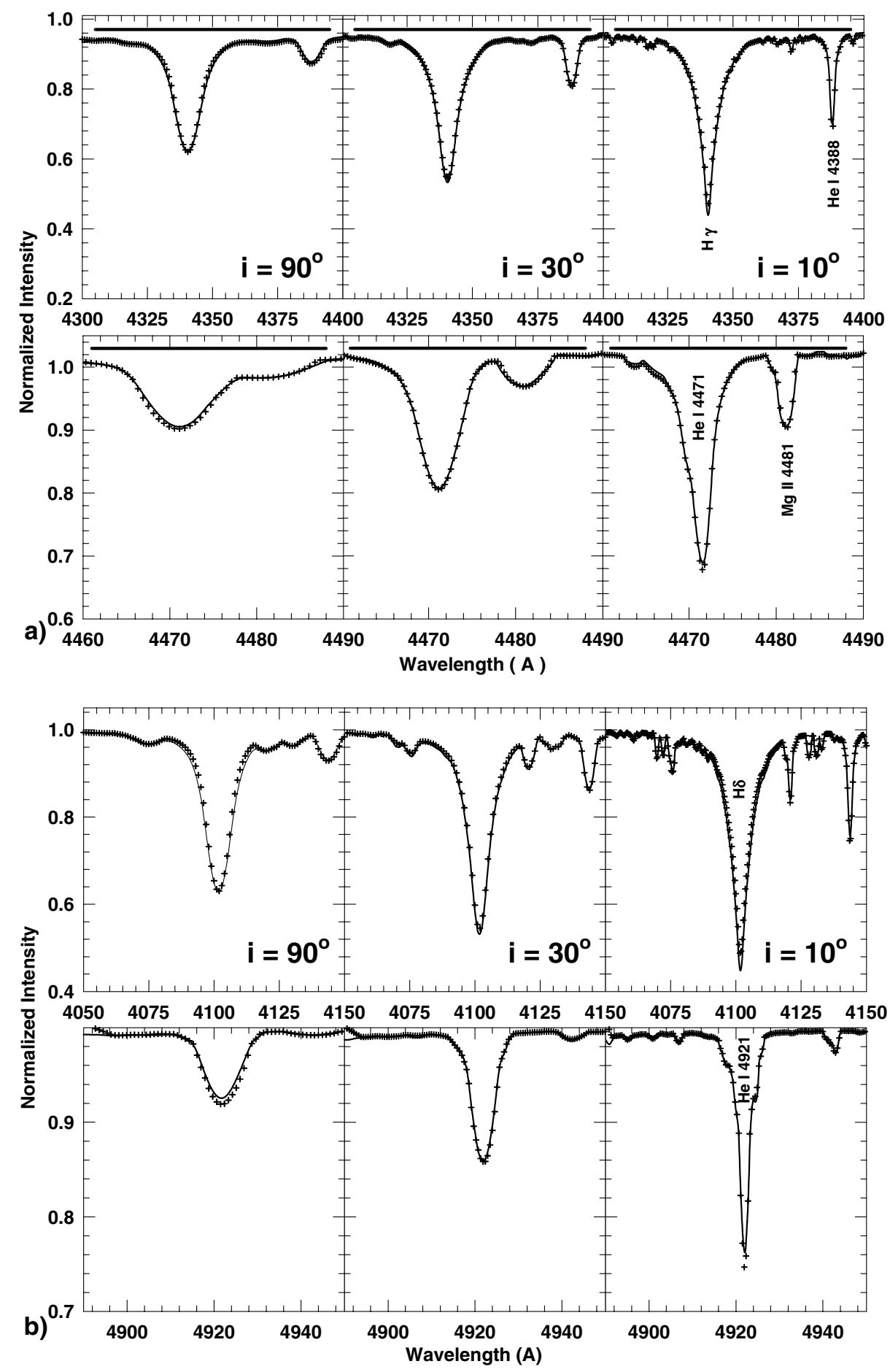

Fig. 9. Comparison between spectra computed using pnrc (full line) and apparent parameters (crosses). Pnrc spectra were computed for $T_{\mathrm{eff}}^{0}=20000 \mathrm{~K}, \log g_{0}=4.0$, and $\Omega / \Omega_{\mathrm{c}}=0.99$. Three different inclination angles were considered. The values of the apparent parameters for each case are given in Table 7.

different $V \sin i$ values $\left(150 \mathrm{~km} \mathrm{~s}^{-1}\right.$ and $\left.200 \mathrm{~km} \mathrm{~s}^{-1}\right)$. The adopted fundamental parameters are listed in Table 6 . This kind of modeling shows that in early B-type stars C II is expected to be underestimated when use is made of a planeparallel model atmosphere for fast rotators seen equator-on. Nevertheless, these models can still be used when stars are seen at low inclinations. The abundance of nitrogen, as derived from various N II lines, is overestimated by a plane-parallel modeling of fast rotators seen at intermediate inclination angles. In fast-rotating B-type stars cooler than $25000 \mathrm{~K}$, oxygen is always overestimated by plane-parallel models when fits of OII spectral lines are done, while it is expected to be somewhat underestimated if one uses the $\lambda 7772$ OI triplet.

More generally, spectral lines preferably formed at the stellar poles (N II, O II, ...) tend to appear stronger when the effects of fast rotation are taken into account (see f.e. O II lines in 

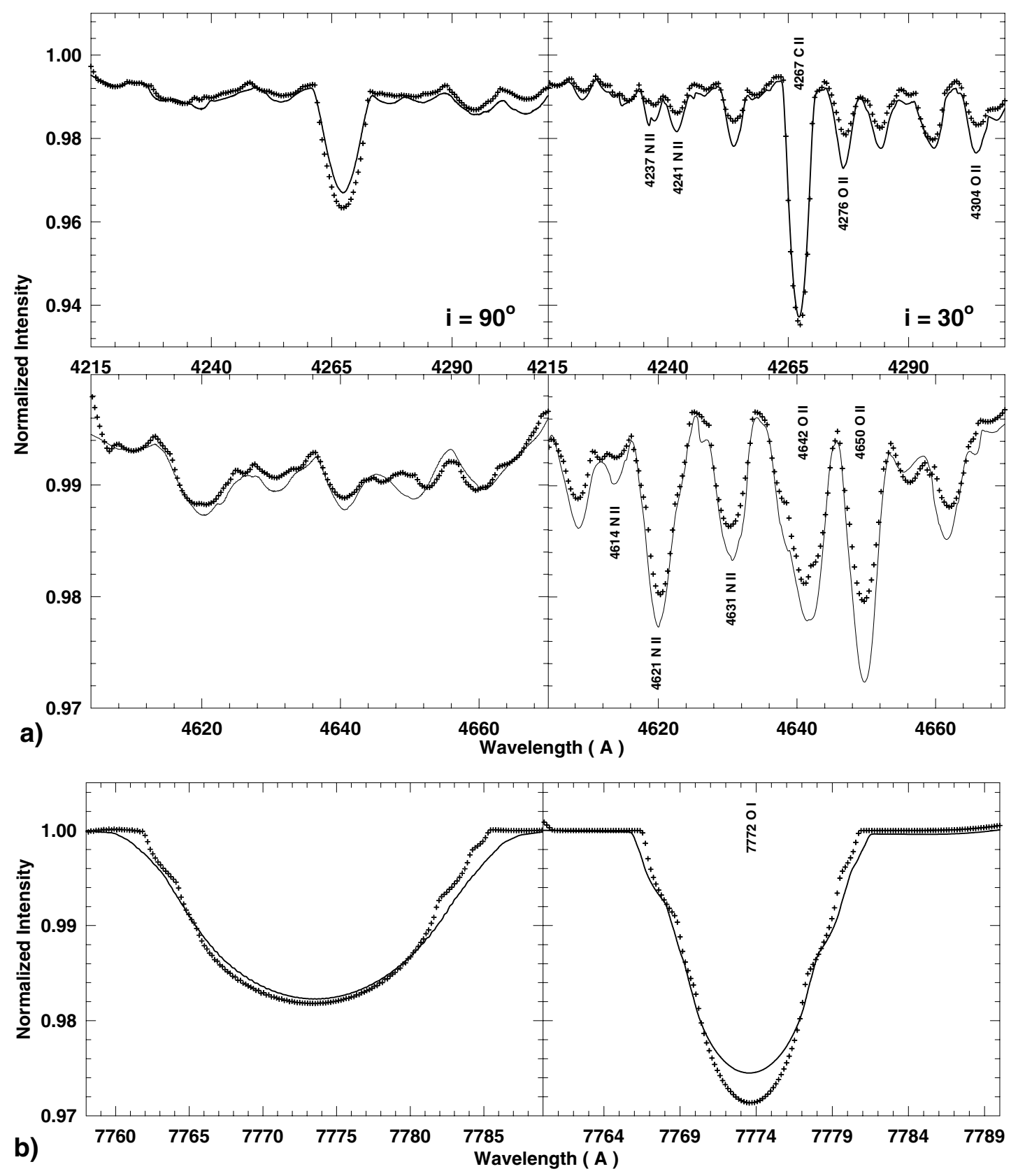

Fig. 10. Comparison between spectra computed using pnrc (full line) and apparent parameters (crosses). Pnrc spectra were computed for $T_{\text {eff }}^{0}=20000 \mathrm{~K}, \log g_{0}=4.0$, and $\Omega / \Omega_{\mathrm{c}}=0.99$. Three different inclination angles were considered. Apparent values for each case are given in Table 7.

panel a of Fig. 10). On the contrary, when their privileged forming region is near the equator, they often appear weaker (see e.g. O I line in panel b) of Fig. 10) but the REFs are also generally smaller. Therefore, REFs on chemical abundance determinations strongly depend on the studied transition, on $V \sin i$, and on the effective temperature of stars. The content of CNO elements in atmospheres of early-type fast rotators, in particular Be stars, will be discussed in a forthcoming paper.

\subsection{Energy distribution}

Since REFs on the stellar continuum are directly proportional to the shape and apparent size of the observed stellar disk, magnitudes are very sensitive to stellar flattening. The GD displayed by the stellar hemisphere facing the observer enhances these effects even more. REFs therefore affect the slope and the absolute level of the emitted fluxes. REFs on the stellar continuum in the visible spectral range have already been widely discussed in many previous works (Collins 1963, 1965, 1966, 1968a,b, 1974; Hardorp \& Strittmatter 1968b, 1972, 1968a; Hardorp \& Scholz 1971; Collins \& Sonneborn 1977; Collins et al. 1991; Maeder \& Peytremann 1970, 1972; Collins \& Smith 1985; Zorec 1986; Pérez Hernández et al. 1999; Townsend et al. 2004). To complete the presentation of rotational effects and because the spatial UV spectral region was less explored in the literature 


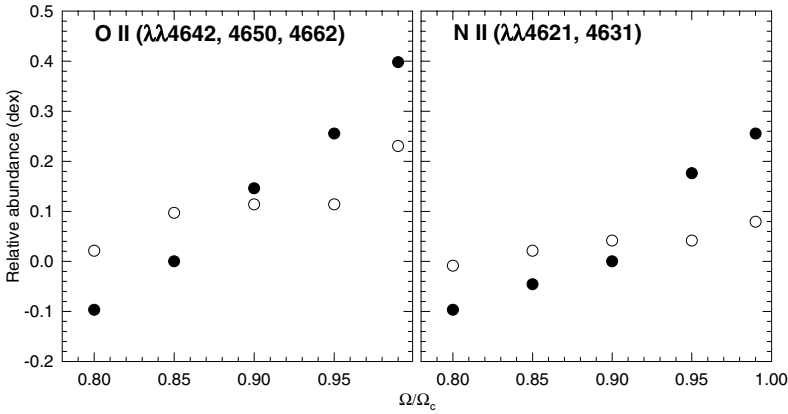

Fig. 11. Abundance overestimation in dex due to stellar flattening and gravitational darkening observed for different values of $\Omega / \Omega_{\mathrm{c}}$ and $V \sin i_{\text {app. }}$ equal to $150 \mathrm{~km} \mathrm{~s}^{-1}$ (open circles) and $200 \mathrm{~km} \mathrm{~s}^{-1}$ (filled circles). Adopted fundamental parameters are listed in Table 6 . Spectral lines used in the abundance estimate are noted in each figure.

Table 6. Apparent ( $\left.T_{\text {eff }}^{\text {app. }}, \log g_{\text {app. }}, V \sin i_{\text {app. }}\right), \operatorname{pnrc}\left(T_{\text {eff }}^{0}, \log g_{0}\right)$ parameters and true $V \sin i$ used to study the effects of stellar flattening on the determination of $\mathrm{CNO}$ abundances (Fig. 11).

\begin{tabular}{|c|c|c|c|c|c|c|c|}
\hline $\begin{array}{l}T_{\text {eff }}^{\text {app. }} \\
(\mathrm{K})\end{array}$ & $\log g_{\text {app. }}$ & $\begin{array}{l}V \sin i_{\text {app. }} \\
\left(\mathrm{km} \mathrm{s}^{-1}\right)\end{array}$ & $\Omega / \Omega_{\mathrm{c}}$ & $\begin{array}{l}T_{\text {eff }}^{0} \\
(\mathrm{~K})\end{array}$ & $\log g_{0}$ & $\begin{array}{l}V \sin i \\
\left(\mathrm{~km} \mathrm{~s}^{-1}\right)\end{array}$ & $\begin{array}{l}i \\
\left(^{\circ}\right)\end{array}$ \\
\hline 19000. & 3.5 & 150 & 0.80 & 19433 & 3.64 & 158 & 38 \\
\hline 19000 . & 3.5 & 150 & 0.85 & 19709 & 3.62 & 160 & 34 \\
\hline 19000. & 3.5 & 150 & 0.90 & 19662 & 3.65 & 161 & 30 \\
\hline 19000. & 3.5 & 150 & 0.95 & 19537 & 3.66 & 164 & 27 \\
\hline 19000. & 3.5 & 150 & 0.99 & 20480 & 3.74 & 170 & 24 \\
\hline 19000. & 3.5 & 200 & 0.80 & 19529 & 3.66 & 207 & 50 \\
\hline 19000. & 3.5 & 200 & 0.85 & 19680 & 3.69 & 210 & 45 \\
\hline 19000 . & 3.5 & 200 & 0.90 & 20070 & 3.71 & 212 & 38 \\
\hline 19000 . & 3.5 & 200 & 0.95 & 20424 & 3.75 & 218 & 35 \\
\hline 19000. & 3.5 & 200 & 0.99 & 20818 & 3.75 & 227 & 32 \\
\hline
\end{tabular}

(Kodaira \& Hoekstra 1979; Llorente de Andres \& Duran 1979), in Fig. 12 we show the comparison between spatial UV energy distribution computed with FASTROT using true and apparent parameters for $\Omega / \Omega_{\mathrm{c}}=0.99$ and different inclination angles. The values of parameters used in each calculation are given in Table 7. Sometimes the spatial region of stellar energy distributions is used to compare apparent diameters or to infer distances (Frémat et al. 2002). According to estimates shown in Fig. 12 differences up to $70 \%$ are expected in these quantities if they concern early type fast rotators.

\section{Discussion}

In the present discussion of the effects of fast rotation on the spectra emitted by early-type stars, we neglected second-order effects due to radiative fluxes related to the latitudinal gradient of the local effective temperature. They might not be entirely negligible (Hadrava 1992), in particular for very fast rotators, where the equatorial gravity $\lim _{\omega \rightarrow 1} g(\mathrm{eq}) \rightarrow 0$. It is expected that their effect may reduce the GD somewhat.

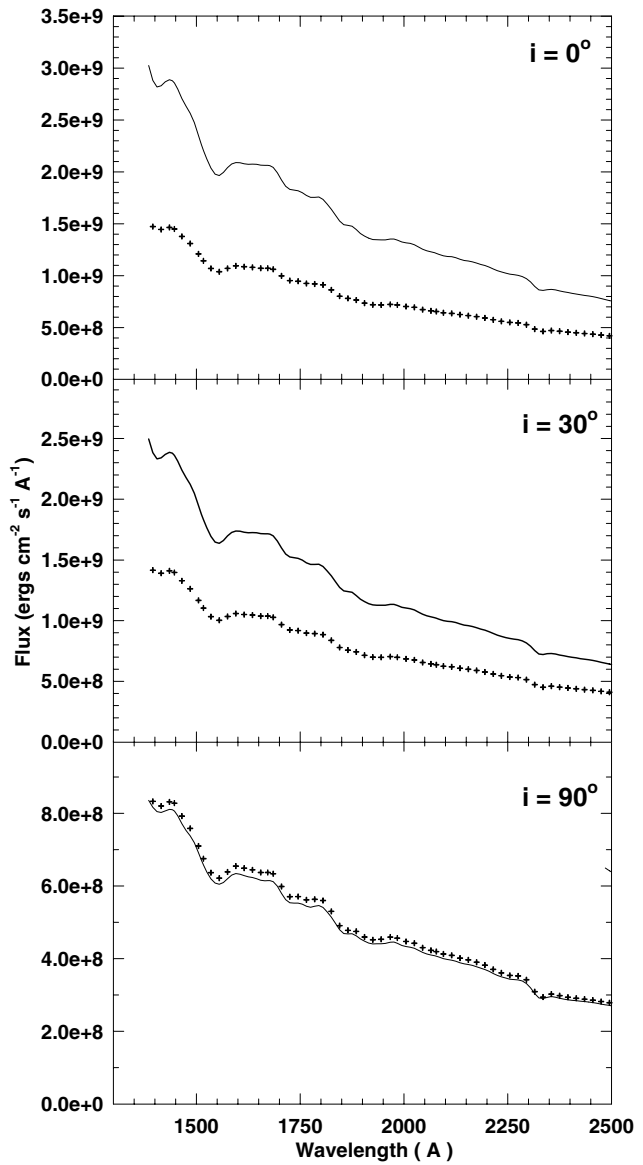

Fig. 12. Comparison between spectra computed using pnrc (full line) and apparent parameters (crosses). Pnrc spectra were computed for $T_{\text {eff }}^{0}=20000 \mathrm{~K}, \log g_{0}=4.0$, and $\Omega / \Omega_{\mathrm{c}}=0.99$. Three different inclination angles were considered. Apparent values for each case are given in Table 7.

Table 7. Apparent and pnrc fundamental parameters used in Figs. 9 and 10 at $\Omega / \Omega_{\mathrm{c}}=0.99$.

\begin{tabular}{rrrrrrr}
\hline \hline$i$ & $T_{\text {eff }}^{0}$ & $\log g_{0}$ & $V \sin i$ & $T_{\text {eff }}^{\text {app. }}$ & $\log g_{\text {app. }}$ & $V \sin i_{\text {app. }}$ \\
\hline 0 & 20000 & 4.0 & 0 & 18675 & 3.83 & 0 \\
30 & 20000 & 4.0 & 231 & 18462 & 3.75 & 203 \\
90 & 20000 & 4.0 & 463 & 16249 & 3.28 & 382 \\
\hline
\end{tabular}

\subsection{Comparison with other recent calculations}

To test our code, we compare the He I $\lambda 4471$ line profile computed with FASTROT and BRUCE (Townsend et al. 2004) in Fig. 13. The same model atmosphere grids were used assuming near critical rotation $\Omega / \Omega_{\mathrm{c}}=0.99\left(V_{\mathrm{e}} / V_{\mathrm{c}}=0.97\right)$ and inclination $i=90^{\circ}$. Relations (5) and (6) were combined to find what pnrc effective temperature is implied by the $T_{\text {polar }}$ value we used as entry parameter in BRUCE. This procedure leads to an almost identical gravitational darkening and to identical line profiles as shown in Fig. 13. We made several comparisons of FASTROT and BRUCE results of the type shown in Fig. 13. Although our integration algorithm is different and outnumbers the equivalent elementary surface elements of BRUCE, quite similar results are obtained. The only difference between 


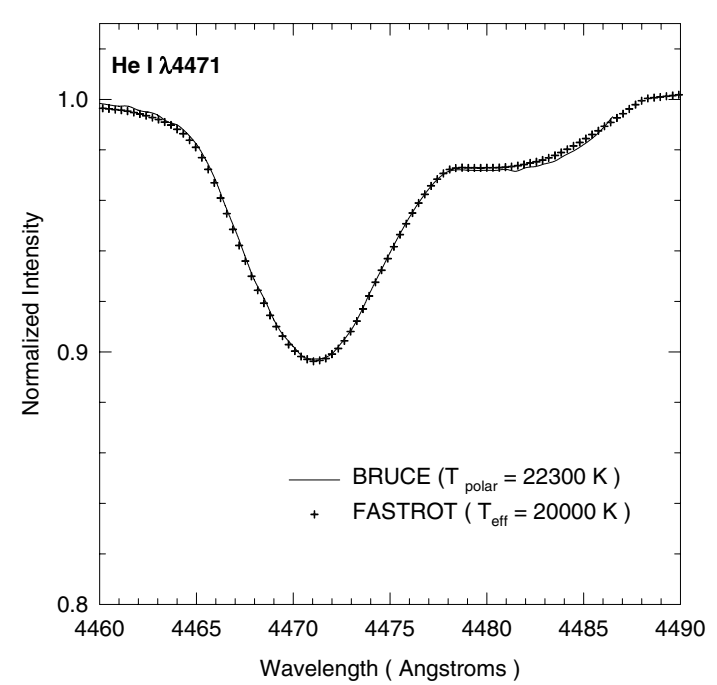

Fig. 13. Comparison between He I $\lambda 4471$ line profiles computed with BRUCE (full line, Townsend et al. 2004) and with FASTROT (crosses). Adopted input parameters in FASTROT are: $T_{\mathrm{eff}}^{0}=20000 \mathrm{~K}$, $\log g_{0}=4.0, \Omega / \Omega_{\mathrm{c}}=0.99$, and $V \sin i=463 \mathrm{~km} \mathrm{~s}^{-1}$ (i.e.: polar temperature $=22300 \mathrm{~K}$ and polar radius $=4.03 R_{\odot}$ ). Adopted input parameters in BRUCE are: polar temperature $=22300 \mathrm{~K}$, polar radius $=4.03 R_{\odot}$, inclination angle $=90^{\circ}$, and equatorial velocity $=463 \mathrm{~km} \mathrm{~s}^{-1}$.

both approaches relies on the adopted fundamental parameters. In FASTROT a unique set of pnrc $T_{\mathrm{eff}}^{0}$ and mass $M$ is used for the whole series of $\Omega$ values, while BRUCE does allow us to specify the temperature distribution in 2 possible ways: by fixing $T_{\text {polar }}$ or by fixing the bolometric luminosity (see Eq. (3) in Townsend et al. 2004).

\subsection{Implications for the study of Be stars}

It has been pointed out by Stoeckley (1968) and Townsend et al. (2004) that the FWHM of the $\lambda 4471 \mathrm{He}$ I line can converge to a constant value at high $V \sin i$ when the stellar angular velocity approaches the critical rotation, which means that the $V \sin i$ values of fast rotators, in particular those of Be stars, can be underestimated. Nevertheless, the implication for the measurements of $V \sin i$ in real cases has still to be explored. Our comparisons between true and apparent values obtained using the fitting procedure (CASE A Table 3), which mimic real cases of $V \sin i$ determination, show that there is progressive lowering of the $V \sin i$ for increasing rotational rates. We also see that this $V \sin i$ underestimation does not necessarily imply a saturation of the estimated projected rotation velocity, even where there is critical rotation (see Fig. 6).

It is interesting then to revisit the most probable value of the $\Omega / \Omega_{\mathrm{c}}$ rate of Be stars if the REFs are taken into account. Chauville et al. (2001) have studied a sample of 116 Be stars and determined their $V \sin i_{\text {app. }}$ parameter without taking the REFs into account. They used, however, Stoeckley \& Mihalas' (1973) calculations of rotationally broadened lines, which consider the wavelength-dependent limb-darkening within the line profiles is considered and which ensures that there is no systematic underestimation of rotational parameters, an effect discussed by Collins \& Truax (1995). Chauville et al. (2001) have thus concluded that on average Be stars rotate at $\Omega / \Omega_{\mathrm{c}} \sim 0.8$. We studied these stars again, along with some others, and carefully determined their apparent fundamental parameters by fitting their spectra with model atmospheres without rotation. We adopted the apparent $V \sin i$ determined by Chauville et al. (2001), in order to avoid the misdetermination of this quantity produced by the Fourier method which uses a constant limb-darkening coefficient in the frequencies over the line profile. Using our GRIDB of synthetic spectra, we determined the pnrc fundamental parameters of the program objects, in particular their $V \sin i$. These parameters were calculated for an average $\Omega / \Omega_{\mathrm{c}}$ rate, which was the same for all stars and whose value was iterated. The iteration started first by assuming $\Omega / \Omega_{\mathrm{c}}=0.8$. At each step we corrected the apparent fundamental parameters in order to obtain the pnrc ones and thereby redetermined the ratios $V \sin i / V_{\mathrm{c}}$ of each star and studied its distribution. Each time we derived the average of $\Omega / \Omega_{\mathrm{c}}$ and its mode from the distribution, i.e. the most frequent or probable value of $\Omega / \Omega_{\mathrm{c}}$. These quantities were estimated in two ways: a) using the same method as Chauville et al. (2001) and b) determining the average and the mode of $V / V_{\mathrm{c}}$ by correcting the distribution of $V \sin i / V_{\mathrm{c}}$ for the $\sin i$ effect. Since the mode of a given distribution depends on at least its first four moments, where errors increase with the order of the moment, we iterated the average $\Omega / \Omega_{\mathrm{c}}$. We also started the iteration assuming all stars rotate at $\Omega / \Omega_{\mathrm{c}}=1.0$. We note that each apparent effective temperature, gravity, and projected rotational velocity was considered with its $2 \sigma$ error. The interval $\left(X-2 \sigma_{X}, X+2 \sigma_{X}\right)$ corresponding to each apparent $X$ parameter was divided into eight parts, so that we had 9 entries for each independent parameter. This produced $9^{3}$ determinations of each corresponding pnrc $X$ parameter or true $V \sin i$. Since each distribution of the obtained pnrc parameters is not symmetrical, we adopted its mode to represent the corresponding most probable solution of the sought pnrc parameter.

Both iterations ended at the same values of the average $\Omega / \Omega_{\mathrm{c}}$ and the mode:

$\overline{\Omega / \Omega_{\mathrm{c}}}=0.85_{-0.04}^{+0.06} \quad\left(\Omega / \Omega_{\mathrm{c}}\right)_{\text {mode }}=0.88_{-0.04}^{+0.06}$.

Table 8 summarizes the characteristics of iterations. Moments $\mu_{\mathrm{n}}$ are for $V / V_{\mathrm{c}}$ distributions. The program stars and their fundamental parameters are given in Table 9. In this table the apparent effective temperature, surface gravity and rotational parameter are given with their respective $1 \sigma$ errors. The modes of the corresponding pnrc parameter and of the corrected $V \sin i$ are also listed, as well as the critical equatorial velocity, the estimated inclination angle, and the $1 \sigma$ dispersion of the respective $9^{3}$ determinations for each fundamental parameter.

From the rates displayed in Eq. (8) we see that critical rotation among Be stars is reachable only by a fraction of objects between $2 \sigma$ and $3 \sigma$ intervals from $\left(\Omega / \Omega_{\mathrm{c}}\right)_{\text {mode }}$. While the difference between apparent and true $V \sin i$ values seems to be significant in some cases, the increase in $\Omega / \Omega_{\mathrm{c}}$ is not so high, as compared to the ratio obtained by Chauville et al. (2001) with apparent $V \sin i$ parameters. This is simply due to the need to also consider the increase from apparent to pnrc values in the stellar fundamental parameters. These identify objects that are more massive and less evolved than expected from the 
Table 8. Iteration of $\overline{\Omega / \Omega_{\mathrm{c}}}$ and $\left(\Omega / \Omega_{\mathrm{c}}\right)_{\text {mode }}$.

\begin{tabular}{lcccccccc}
\hline \hline & & \multicolumn{9}{c}{ modes } \\
$\Omega / \Omega_{\mathrm{c}}$ & $\overline{V / V_{\mathrm{c}}}$ & $\overline{\Omega / \Omega_{\mathrm{c}}}$ & $V / V_{\mathrm{c}}$ & $\Omega / \Omega_{\mathrm{c}}$ & $\mu_{2}$ & $\mu_{3}$ & $\mu_{4}$ \\
\hline 0.800 & 0.708 & 0.842 & 0.756 & 0.879 & 0.507 & 0.369 & 0.271 \\
0.842 & 0.712 & 0.846 & 0.753 & 0.878 & 0.513 & 0.375 & 0.277 \\
0.846 & 0.713 & 0.847 & 0.746 & 0.872 & 0.515 & 0.376 & 0.277 \\
0.847 & 0.713 & 0.847 & 0.751 & 0.876 & 0.515 & 0.376 & 0.278 \\
\hline 1.000 & 0.782 & 0.898 & 0.726 & 0.854 & 0.627 & 0.513 & 0.428 \\
0.898 & 0.725 & 0.856 & 0.673 & 0.814 & 0.533 & 0.397 & 0.300 \\
0.856 & 0.715 & 0.848 & 0.733 & 0.862 & 0.518 & 0.379 & 0.281 \\
0.848 & 0.713 & 0.846 & 0.732 & 0.861 & 0.514 & 0.376 & 0.278 \\
0.846 & 0.713 & 0.847 & 0.751 & 0.876 & 0.514 & 0.375 & 0.277 \\
\hline
\end{tabular}

apparent parameters. Such increase also implies a significant augmentation of the equatorial critical velocity $V_{\mathrm{c}}$, which is barely compensated by the respective true $V \sin i$ value.

\subsubsection{Comments on the solutions obtained}

We recall that the values of parameters displayed in Table 9 are "modes" of the respective distributions of $9^{3}$ possible solutions. They can be considered as representing the most probable configuration of fundamental parameters for the rotating star, as they are perceived from the entry set of apparent parameters with their uncertainties, seen through the models calculated with FASTROT used to interpret them. They look like sort of independent "determinations" which also sometimes seem to underestimate the effects due to fast rotation. Nevertheless, if we used only the apparent entry parameters without their uncertainties, the unique translation into pnrc quantities that comes out would lead in many cases to $\Delta T_{\text {eff }}, \Delta \log g$, and $\Delta V \sin i$ differences between pnrc and apparent values that are higher than those issued from the values in Table 9. We calculated the $\overline{\Omega / \Omega_{\mathrm{c}}}$ and $\left(\Omega / \Omega_{\mathrm{c}}\right)_{\text {mode }}$ using these simplified estimates of pnrc parameters and true $V \sin i$, as well as the same iteration procedure for $\Omega / \Omega_{\mathrm{c}}$. We thus obtained $\overline{\Omega / \Omega_{\mathrm{c}}}=0.83$ near the value put forward in Zorec et al. (2003) and $\left(\Omega / \Omega_{\mathrm{c}}\right)_{\text {mode }}=0.88$. This small increase in the $\Omega / \Omega_{\mathrm{c}}$, as compared to the value obtained by Chauville et al. (2001), is entirely ascribed to the higher $V_{\mathrm{c}}$ values implied by the apparent $\rightarrow$ pnrc transformation of fundamental parameters.

\subsection{2. pnrc and actual fundamental parameters}

In this paper, we have studied the relation between the apparent parameters and those that should represent the actual stellar mass and its evolutionary stage. The translation obtained into pnrc parameters was performed with the help of evolutionary tracks calculated for non-rotating stars (Schaller et al. 1992). However, evolutionary tracks of rotating objects are somewhat different (Heger \& Langer 2000; Meynet \& Maeder 2000). Depending on the case, they may produce slightly different mass estimates. These estimates are those which we might finally consider as a better approach to the actual stellar mass.
Nevertheless, these differences are small and do not modify significantly the results obtained in the preceding sections. Their ins and outs are out of the scope of the present work and will be widely discussed in another contribution.

\section{Conclusions}

In this paper we present the calculation code FASTROT to calculate the effects of fast rotation on the fundamental parameters of early-type stars. Particular attention is payed to represent hydrogen Balmer, He I, and Mg II lines in the blue spectral region, which are currently used to infer the fundamental parameters of early-type stars from spectroscopic data. We also calculated a number of $\mathrm{C}, \mathrm{N}$, and $\mathrm{O}$ lines in the visible spectral range, which enter the CNO abundance determination.

We calculated synthetic spectra with classical planeparallel non-LTE model atmospheres using apparent fundamental parameters derived from $\mathrm{H} \gamma$, He I 4471, and Mg II 4481 lines and compared their predictions for other spectral regions of rotating stars. We noted that the above apparent fundamental also reliably reproduces the remaining Balmer and He I lines in the visual. However, discrepant fits are expected for CNO lines. The noted discrepancies may thus lead to systematic overestimates of the $\mathrm{N}$ abundances, while the $\mathrm{C}$ abundances can be underestimated. The $\mathrm{O}$ abundance depends on the lines used. They are sensitive to local formation conditions and reflect preferences either on the polar or equatorial formation regions. $\mathrm{O}$ abundances can then be under- or overestimated, depending on the lines used and depending on the stellar configuration or inclination angle.

The fastest rotation in the main sequence are probably Be stars. Their strong gravitational darkening can then lead to sensitive $V \sin i$ underestimations, which in turn may imply that the hitherto undercritical rotation of these stars can be argued. We payed close attention then to the determination of the fundamental parameters of fast rotators, in particular to $V \sin i$. We thus found that although the classical $V \sin i$ parameter do underestimate the stellar equatorial velocity of fast rotators, the fact that the pnrc stellar mass is higher and the evolutionary stage lower than expected from apparent quantities, means that in most cases the increase in the estimate of $V \sin i$ does not compensate the new or pnrc $V_{\mathrm{c}}$ value. The study of a sample of $130 \mathrm{Be}$ stars leads thus to the most probable ratio $V_{\mathrm{e}} / V_{\mathrm{c}} \simeq$ 0.75 , or $\Omega / \Omega_{\mathrm{c}} \simeq 0.88$, which still implies average undercritical surface rotation.

Acknowledgements. We are grateful to the referee Dr. I. D. Howarth and to Dr. R. Townsend, as their comments and suggestions helped to improve the presentation of our results. Y.F. thanks Dr. P. Lampens for hosting him at the Royal Observatory of Belgium and acknowledges funding from the Belgian "Diensten van de Eerste Minister Federale Diensten voor Wetenschappelijke, Technische en Culturele Aangelegenheden" (Research project MO/33/007).

\section{References}

Barnard, A. J., Cooper, J., \& Shamey, L. J. 1969, A\&A, 1, 28

Bodenheimer, P. 1971, ApJ, 167, 153

Castelli, F., \& Kurucz, R. L. 2003, IAU Symp., 210, 20 
Chauville, J., Zorec, J., Ballereau, D., et al. 2001, A\&A, 378, 861

Claret, A. 1998, A\&A, 335, 647

Claret, A. 2000, A\&A, 359, 289

Clement, M. J. 1979, ApJ, 230, 230

Collins, G. W. 1963, ApJ, 138, 1134

Collins, G. W. 1965, ApJ, 142, 265

Collins, G. W. 1966, ApJ, 146, 914

Collins, G. W. 1968a, ApJ, 151, 217

Collins, G. W. 1968b, ApJ, 152, 847

Collins, G. W. 1974, ApJ, 191, 157

Collins, G. W., \& Smith, R. C. 1985, MNRAS, 213, 519

Collins, G. W., \& Sonneborn, G. H. 1977, ApJS, 34, 41

Collins, G. W., \& Truax, R. J. 1995, ApJ, 439, 860

Collins, G. W., Truax, R. J., \& Cranmer, S. R. 1991, ApJS, 77, 541

Cunto, W., Mendoza, C., Ochsenbein, F., \& Zeippen, C. J. 1993, A\&A, 275, L5

Didelon, P. 1982, A\&AS, 50, 199

Endal, A. S., \& Sofia, S. 1976, ApJ, 210, 184

Endal, A. S., \& Sofia, S. 1979, ApJ, 232, 531

Frémat, Y., Zorec, J., Hubert, A.-M., et al. 2002, A\&A, 385, 986

Gray, R. O., \& Corbally, C. J. 1994, AJ, 107, 742

Hadrava, P. 1992, A\&A, 256, 519

Hardorp, J., \& Scholz, M. 1971, A\&A, 13, 353

Hardorp, J., \& Strittmatter, P. A. 1968a, ApJ, 153, 465

Hardorp, J., \& Strittmatter, P. A. 1968b, ApJ, 151, 1057

Hardorp, J., \& Strittmatter, P. A. 1972, A\&A, 17, 161

Heger, A., \& Langer, N. 2000, ApJ, 544, 1016

Hubeny, I., \& Lanz, T. 1995, ApJ, 439, 875

Kodaira, K., \& Hoekstra, R. 1979, A\&A, 78, 292

Kurucz, R. L. 1993, Kurucz CD-ROM No. 13. Cambridge, Mass.: Smithsonian Astrophysical Observatory

Lanz, T., \& Hubeny, I. 2003, ApJS, 146, 417

Leckrone, D. S. 1971, A\&A, 11, 387

Llorente de Andres, F., \& Duran, C. M. 1979, A\&A, 72, 318

Lucy, L. B. 1967, Z. Astrophys., 65, 89

Maeder, A., \& Meynet, G. 1996, A\&A, 313, 140

Maeder, A., \& Meynet, G. 2000, A\&A, 361, 159
Maeder, A., \& Peytremann, E. 1970, A\&A, 7, 120

Maeder, A., \& Peytremann, E. 1972, A\&A, 21, 279

Meynet, G., \& Maeder, A. 2000, A\&A, 361, 101

Mihalas, D., Barnard, A. J., Cooper, J., \& Smith, E. W. 1974, ApJ, 190, 315

Moss, D., \& Smith, R. C. 1982, Rep. Progr. Phys., 44, 831

Pérez Hernández, F., Claret, A., Hernández, M. M., \& Michel, E. 1999, A\&A, 346, 586

Pinsonneault, M. H., Deliyannis, C. P., \& Demarque, P. 1991, ApJ, 367, 239

Reiners, A. 2003, A\&A, 408, 707

Sackmann, I. J. 1970, A\&A, 8, 76

Schaller, G., Schaerer, D., Meynet, G., \& Maeder, A. 1992, A\&AS, 96,269

Sigut, T. A. A. 1996, ApJ, 473, 452

Slettebak, A., Collins, G. W., Parkinson, T. D., Boyce, P. B., \& White, N. M. 1975, ApJS, 29, 137

Smith, C. R., \& Worley, R. 1974, MNRAS, 167, 199

Stoeckley, T. R. 1968, MNRAS, 140, 149

Tassoul, J. 1978, Theory of rotating stars, Princeton Series in Astrophysics (Princeton: University Press)

Townsend, R. H. D., Owocki, S. P., \& Howarth, I. D. 2004, MNRAS, 350,189

Tuominen, J. 1972, Astrophys. Lett., 10, 175

Varosi, F., Lanz, T., deKoter, A., et al. 1995, ftp://idlastro.gsfc.nasa.gov/pub/contrib/ varosi/modion

Vidal, C. R., Cooper, J., \& Smith, E. W. 1973, ApJS, 25, 37

Zahn, J.-P. 1992, A\&A, 265, 115

Zeipel, H. V. 1924, MNRAS, 84, 665

Zorec, J. 1986, Ph.D. Thesis: Structure et rotation différentielle dans les étoiles B avec et sans émission, Paris: Université VII

Zorec, J., Frémat, Y., Ballereau, D., et al. 2003, in SF2A-2003: Semaine de l'Astrophysique Française, 617

Zorec, J., Mochkovitch, R., \& Divan, L. 1988, Academie des Sciences Paris Comptes Rendus Ser. Sci. Math., 306, 1265 


\section{Online Material}


Y. Frémat et al.: Effects of fast rotation, Online Material $p 2$

Table 9. Apparent and pnrc fundamental parameters of the studied stars.

\begin{tabular}{|c|c|c|c|c|c|c|c|c|}
\hline \multirow[b]{2}{*}{ HD } & \multicolumn{3}{|c|}{ Apparent parameters } & \multicolumn{5}{|c|}{ Mode of the pnrc parameters } \\
\hline & $T_{\text {eff }} \pm \epsilon$ & $\log g \pm \epsilon$ & $V \sin i \pm \epsilon$ & $T_{\text {eff }} \pm 1 \sigma$ & $\log g \pm 1 \sigma$ & $V \sin i \pm 1 \sigma$ & $V_{\mathrm{c}} \pm 1 \sigma$ & $i^{o} \pm 1 \sigma$ \\
\hline 144 & $11956 \pm 324$ & $3.383 \pm 0.055$ & $125 \pm 07$ & $12206 \pm 414$ & $3.403 \pm 0.067$ & $132 \pm 08$ & $309 \pm 20$ & $33.9 \pm 1.2$ \\
\hline 4180 & $14438 \pm 272$ & $3.284 \pm 0.041$ & $195 \pm 10$ & $15584 \pm 426$ & $3.399 \pm 0.062$ & $208 \pm 13$ & $332 \pm 21$ & $55.7 \pm 3.2$ \\
\hline 5394 & $26431 \pm 618$ & $3.800 \pm 0.070$ & $432 \pm 28$ & $30001 \pm 624$ & $3.990 \pm 0.065$ & $441 \pm 27$ & $577 \pm 36$ & $76.4 \pm 2.7$ \\
\hline 6811 & $12478 \pm 444$ & $3.092 \pm 0.068$ & $85 \pm 05$ & $12578 \pm 481$ & $3.110 \pm 0.073$ & $93 \pm 06$ & $274 \pm 19$ & $26.5 \pm 1.0$ \\
\hline 10516 & $25556 \pm 659$ & $3.899 \pm 0.078$ & $440 \pm 30$ & $29031 \pm 751$ & $4.168 \pm 0.087$ & $462 \pm 33$ & $590 \pm 42$ & $72.5 \pm 3.9$ \\
\hline 11606 & $19484 \pm 297$ & $3.653 \pm 0.037$ & $280 \pm 14$ & $21422 \pm 439$ & $3.774 \pm 0.055$ & $286 \pm 16$ & $446 \pm 26$ & $58.2 \pm 3.5$ \\
\hline 18552 & $12715 \pm 284$ & $3.456 \pm 0.048$ & $286 \pm 16$ & $14646 \pm 244$ & $3.776 \pm 0.041$ & $292 \pm 14$ & $369 \pm 19$ & $75.2 \pm 5.0$ \\
\hline 19243 & $21243 \pm 388$ & $3.270 \pm 0.043$ & $160 \pm 08$ & $22752 \pm 409$ & $3.297 \pm 0.043$ & $165 \pm 09$ & $387 \pm 21$ & $36.2 \pm 1.3$ \\
\hline 20336 & $18684 \pm 517$ & $3.865 \pm 0.072$ & $328 \pm 21$ & $20843 \pm 558$ & $4.050 \pm 0.076$ & $341 \pm 23$ & $487 \pm 32$ & $66.7 \pm 4.0$ \\
\hline 22192 & $15767 \pm 509$ & $3.465 \pm 0.074$ & $275 \pm 19$ & $17690 \pm 299$ & $3.763 \pm 0.042$ & $295 \pm 15$ & $397 \pm 20$ & $75.0 \pm 3.1$ \\
\hline 22780 & $13299 \pm 284$ & $3.381 \pm 0.046$ & $285 \pm 15$ & $15686 \pm 443$ & $3.572 \pm 0.066$ & $297 \pm 18$ & $366 \pm 23$ & $90.0 \pm 1.0$ \\
\hline 23016 & $12230 \pm 241$ & $3.922 \pm 0.047$ & $235 \pm 12$ & $13420 \pm 249$ & $4.012 \pm 0.047$ & $242 \pm 12$ & $410 \pm 21$ & $48.7 \pm 2.6$ \\
\hline 23302 & $12754 \pm 504$ & $3.368 \pm 0.078$ & $170 \pm 12$ & $13484 \pm 293$ & $3.412 \pm 0.047$ & $181 \pm 10$ & $320 \pm 18$ & $46.8 \pm 1.6$ \\
\hline 23480 & $13691 \pm 481$ & $3.629 \pm 0.076$ & $240 \pm 16$ & $14828 \pm 402$ & $3.792 \pm 0.064$ & $254 \pm 15$ & $391 \pm 24$ & $57.3 \pm 3.3$ \\
\hline 23552 & $12711 \pm 416$ & $3.764 \pm 0.070$ & $220 \pm 14$ & $13708 \pm 239$ & $3.807 \pm 0.043$ & $230 \pm 12$ & $387 \pm 20$ & $52.0 \pm 2.2$ \\
\hline 23630 & $12258 \pm 505$ & $3.047 \pm 0.076$ & $140 \pm 10$ & $12885 \pm 292$ & $3.095 \pm 0.045$ & $149 \pm 08$ & $274 \pm 15$ & $44.6 \pm 1.6$ \\
\hline 23862 & $12106 \pm 272$ & $3.937 \pm 0.052$ & $286 \pm 16$ & $13436 \pm 257$ & $4.197 \pm 0.050$ & $290 \pm 15$ & $420 \pm 22$ & $65.3 \pm 3.9$ \\
\hline 24534 & $25191 \pm 513$ & $3.618 \pm 0.056$ & $293 \pm 17$ & $26831 \pm 699$ & $3.854 \pm 0.081$ & $294 \pm 20$ & $480 \pm 34$ & $54.7 \pm 2.6$ \\
\hline 25940 & $16158 \pm 582$ & $3.572 \pm 0.084$ & $197 \pm 14$ & $17593 \pm 338$ & $3.789 \pm 0.049$ & $220 \pm 13$ & $386 \pm 21$ & $38.3 \pm 4.5$ \\
\hline 28497 & $26724 \pm 427$ & $4.200 \pm 0.046$ & $335 \pm 17$ & $28101 \pm 738$ & $4.222 \pm 0.088$ & $342 \pm 24$ & $631 \pm 45$ & $45.4 \pm 2.0$ \\
\hline 30076 & $20488 \pm 330$ & $3.772 \pm 0.041$ & $213 \pm 11$ & $21311 \pm 397$ & $3.792 \pm 0.049$ & $225 \pm 12$ & $456 \pm 25$ & $38.4 \pm 1.7$ \\
\hline 32343 & $16131 \pm 514$ & $3.798 \pm 0.077$ & $95 \pm 06$ & $16121 \pm 430$ & $3.790 \pm 0.065$ & $103 \pm 06$ & $410 \pm 26$ & $19.7 \pm 0.8$ \\
\hline 33328 & $21137 \pm 599$ & $3.447 \pm 0.075$ & $318 \pm 22$ & $24340 \pm 644$ & $3.608 \pm 0.076$ & $333 \pm 23$ & $440 \pm 30$ & $73.0 \pm 2.4$ \\
\hline 35411 & $23652 \pm 510$ & $3.711 \pm 0.059$ & $170 \pm 10$ & $25138 \pm 441$ & $3.719 \pm 0.046$ & $174 \pm 09$ & $484 \pm 26$ & $29.0 \pm 1.3$ \\
\hline 35439 & $22134 \pm 665$ & $3.920 \pm 0.087$ & $263 \pm 19$ & $23547 \pm 364$ & $3.948 \pm 0.041$ & $266 \pm 13$ & $513 \pm 26$ & $42.2 \pm 2.3$ \\
\hline 36576 & $22618 \pm 508$ & $3.804 \pm 0.062$ & $265 \pm 16$ & $24502 \pm 399$ & $3.839 \pm 0.043$ & $266 \pm 13$ & $487 \pm 25$ & $46.3 \pm 2.2$ \\
\hline 37202 & $19310 \pm 550$ & $3.732 \pm 0.075$ & $310 \pm 7$ & $21460 \pm 420$ & $3.910 \pm 0.060$ & $326 \pm 7$ & $466 \pm 13$ & $66.0 \pm 4.1$ \\
\hline 37657 & $17951 \pm 542$ & $3.659 \pm 0.076$ & $198 \pm 13$ & $19275 \pm 301$ & $3.686 \pm 0.039$ & $209 \pm 10$ & $427 \pm 21$ & $41.9 \pm 1.9$ \\
\hline 37795 & $12963 \pm 203$ & $3.517 \pm 0.036$ & $180 \pm 09$ & $13695 \pm 437$ & $3.559 \pm 0.069$ & $192 \pm 12$ & $355 \pm 23$ & $44.9 \pm 2.1$ \\
\hline 37967 & $16543 \pm 264$ & $3.850 \pm 0.041$ & $210 \pm 10$ & $17558 \pm 589$ & $3.873 \pm 0.085$ & $228 \pm 16$ & $455 \pm 33$ & $42.1 \pm 2.1$ \\
\hline 38010 & $25826 \pm 448$ & $4.089 \pm 0.050$ & $370 \pm 20$ & $28015 \pm 555$ & $4.194 \pm 0.061$ & $377 \pm 22$ & $608 \pm 36$ & $53.0 \pm 3.4$ \\
\hline 40978 & $18227 \pm 571$ & $3.576 \pm 0.078$ & $200 \pm 14$ & $19605 \pm 550$ & $3.608 \pm 0.073$ & $211 \pm 14$ & $401 \pm 27$ & $43.3 \pm 1.9$ \\
\hline 41335 & $20902 \pm 610$ & $3.886 \pm 0.081$ & $358 \pm 25$ & $23431 \pm 639$ & $4.076 \pm 0.082$ & $376 \pm 26$ & $520 \pm 36$ & $68.5 \pm 3.8$ \\
\hline 42545 & $15764 \pm 334$ & $3.849 \pm 0.053$ & $285 \pm 16$ & $17522 \pm 309$ & $3.976 \pm 0.046$ & $303 \pm 16$ & $460 \pm 24$ & $60.1 \pm 4.0$ \\
\hline 44458 & $22914 \pm 532$ & $3.600 \pm 0.063$ & $242 \pm 15$ & $24745 \pm 667$ & $3.640 \pm 0.079$ & $254 \pm 18$ & $449 \pm 31$ & $48.0 \pm 2.1$ \\
\hline 45314 & $29160 \pm 467$ & $3.949 \pm 0.044$ & $285 \pm 14$ & $31092 \pm 557$ & $3.968 \pm 0.053$ & $291 \pm 16$ & $594 \pm 33$ & $40.6 \pm 1.9$ \\
\hline 45542 & $13711 \pm 526$ & $3.627 \pm 0.082$ & $217 \pm 15$ & $14651 \pm 229$ & $3.682 \pm 0.038$ & $226 \pm 11$ & $369 \pm 18$ & $52.5 \pm 2.6$ \\
\hline 45725 & $17810 \pm 455$ & $3.895 \pm 0.066$ & $330 \pm 20$ & $19601 \pm 459$ & $4.168 \pm 0.065$ & $345 \pm 21$ & $486 \pm 30$ & $66.6 \pm 3.9$ \\
\hline 45910 & $20659 \pm 484$ & $2.739 \pm 0.052$ & $240 \pm 14$ & $22983 \pm 471$ & $2.992 \pm 0.048$ & $254 \pm 14$ & $342 \pm 20$ & $76.5 \pm 3.5$ \\
\hline 45995 & $21198 \pm 632$ & $3.769 \pm 0.083$ & $255 \pm 18$ & $22674 \pm 423$ & $3.806 \pm 0.050$ & $260 \pm 14$ & $470 \pm 26$ & $46.8 \pm 2.1$ \\
\hline 47054 & $12336 \pm 481$ & $3.538 \pm 0.078$ & $219 \pm 15$ & $13292 \pm 355$ & $3.607 \pm 0.058$ & $227 \pm 13$ & $351 \pm 21$ & $57.2 \pm 2.2$ \\
\hline 48917 & $20341 \pm 435$ & $3.395 \pm 0.053$ & $205 \pm 12$ & $21348 \pm 569$ & $3.438 \pm 0.070$ & $212 \pm 14$ & $389 \pm 26$ & $45.4 \pm 2.0$ \\
\hline 50013 & $24627 \pm 590$ & $4.017 \pm 0.072$ & $243 \pm 15$ & $25790 \pm 713$ & $4.026 \pm 0.087$ & $244 \pm 17$ & $535 \pm 39$ & $37.3 \pm 1.9$ \\
\hline 50083 & $20908 \pm 604$ & $3.678 \pm 0.078$ & $170 \pm 12$ & $21216 \pm 625$ & $3.689 \pm 0.081$ & $176 \pm 12$ & $452 \pm 32$ & $32.5 \pm 1.4$ \\
\hline 53974 & $27808 \pm 759$ & $3.650 \pm 0.085$ & $100 \pm 17$ & $27624 \pm 577$ & $3.649 \pm 0.060$ & $107 \pm 06$ & $490 \pm 30$ & $16.5 \pm 0.6$ \\
\hline 54309 & $20859 \pm 397$ & $3.587 \pm 0.048$ & $195 \pm 10$ & $22235 \pm 543$ & $3.610 \pm 0.066$ & $201 \pm 13$ & $425 \pm 27$ & $38.4 \pm 1.5$ \\
\hline 56014 & $18544 \pm 614$ & $3.287 \pm 0.081$ & $280 \pm 20$ & $21061 \pm 453$ & $3.514 \pm 0.055$ & $290 \pm 17$ & $389 \pm 23$ & $72.6 \pm 4.2$ \\
\hline 56139 & $19537 \pm 331$ & $3.615 \pm 0.042$ & $85 \pm 04$ & $19465 \pm 300$ & $3.612 \pm 0.037$ & $89 \pm 04$ & $403 \pm 20$ & $16.6 \pm 0.7$ \\
\hline 57219 & $19865 \pm 575$ & $3.874 \pm 0.078$ & $80 \pm 05$ & $19759 \pm 486$ & $3.862 \pm 0.066$ & $84 \pm 05$ & $443 \pm 28$ & $14.3 \pm 0.7$ \\
\hline 58050 & $19961 \pm 465$ & $3.934 \pm 0.063$ & $130 \pm 08$ & $19998 \pm 590$ & $3.926 \pm 0.081$ & $136 \pm 09$ & $481 \pm 34$ & $21.9 \pm 1.2$ \\
\hline 58343 & $16531 \pm 409$ & $3.619 \pm 0.059$ & $43 \pm 02$ & $16389 \pm 437$ & $3.611 \pm 0.063$ & $49 \pm 03$ & $377 \pm 24$ & $9.7 \pm 0.3$ \\
\hline 58715 & $11772 \pm 344$ & $3.811 \pm 0.062$ & $230 \pm 14$ & $12769 \pm 395$ & $3.858 \pm 0.068$ & $231 \pm 14$ & $380 \pm 24$ & $54.5 \pm 2.9$ \\
\hline 58978 & $24445 \pm 476$ & $4.153 \pm 0.057$ & $370 \pm 21$ & $26622 \pm 717$ & $4.203 \pm 0.088$ & $375 \pm 26$ & $615 \pm 44$ & $55.2 \pm 3.0$ \\
\hline
\end{tabular}


Y. Frémat et al.: Effects of fast rotation, Online Material p 3

Table 9. continued.

\begin{tabular}{|c|c|c|c|c|c|c|c|c|}
\hline \multirow[b]{2}{*}{ HD } & \multicolumn{3}{|c|}{ Apparent parameters } & \multicolumn{5}{|c|}{ Mode of the pnrc parameters } \\
\hline & $T_{\text {eff }} \pm \epsilon$ & $\log g \pm \epsilon$ & $V \sin i \pm \epsilon$ & $T_{\text {eff }} \pm 1 \sigma$ & $\log g \pm 1 \sigma$ & $V \sin i \pm 1 \sigma$ & $V_{\mathrm{c}} \pm 1 \sigma$ & $i^{o} \pm 1 \sigma$ \\
\hline 60606 & $18030 \pm 332$ & $3.662 \pm 0.045$ & $273 \pm 14$ & $19600 \pm 414$ & $3.783 \pm 0.055$ & $285 \pm 16$ & $441 \pm 25$ & $58.2 \pm 3.6$ \\
\hline 60848 & $27685 \pm 671$ & $4.051 \pm 0.077$ & $247 \pm 16$ & $28491 \pm 630$ & $4.057 \pm 0.070$ & $256 \pm 16$ & $582 \pm 37$ & $35.4 \pm 1.8$ \\
\hline 63462 & $26558 \pm 635$ & $3.598 \pm 0.070$ & $440 \pm 29$ & $30685 \pm 728$ & $3.835 \pm 0.077$ & $514 \pm 31$ & $545 \pm 37$ & $90.0 \pm 1.0$ \\
\hline 65875 & $20205 \pm 532$ & $3.845 \pm 0.071$ & $153 \pm 10$ & $20358 \pm 590$ & $3.844 \pm 0.079$ & $163 \pm 11$ & $447 \pm 31$ & $27.8 \pm 1.2$ \\
\hline 68980 & $25126 \pm 642$ & $3.951 \pm 0.077$ & $145 \pm 09$ & $25172 \pm 492$ & $3.945 \pm 0.056$ & $152 \pm 08$ & $534 \pm 31$ & $22.2 \pm 1.2$ \\
\hline 75311 & $16943 \pm 429$ & $3.846 \pm 0.064$ & $268 \pm 16$ & $18513 \pm 527$ & $4.089 \pm 0.077$ & $283 \pm 19$ & $467 \pm 31$ & $54.2 \pm 4.0$ \\
\hline 77320 & $19555 \pm 488$ & $3.871 \pm 0.067$ & $345 \pm 22$ & $21539 \pm 637$ & $4.015 \pm 0.085$ & $349 \pm 25$ & $501 \pm 36$ & $68.7 \pm 3.6$ \\
\hline 83953 & $15187 \pm 329$ & $3.661 \pm 0.051$ & $260 \pm 14$ & $16616 \pm 273$ & $3.788 \pm 0.041$ & $276 \pm 14$ & $407 \pm 21$ & $61.0 \pm 4.0$ \\
\hline 86612 & $16374 \pm 577$ & $3.580 \pm 0.083$ & $185 \pm 13$ & $17392 \pm 381$ & $3.790 \pm 0.055$ & $206 \pm 12$ & $381 \pm 22$ & $43.3 \pm 4.2$ \\
\hline 88661 & $21527 \pm 384$ & $3.989 \pm 0.049$ & $237 \pm 12$ & $22334 \pm 450$ & $4.003 \pm 0.057$ & $243 \pm 14$ & $511 \pm 29$ & $39.0 \pm 2.1$ \\
\hline 89080 & $11720 \pm 431$ & $3.339 \pm 0.070$ & $240 \pm 16$ & $13275 \pm 251$ & $3.581 \pm 0.043$ & $245 \pm 13$ & $320 \pm 17$ & $70.8 \pm 3.0$ \\
\hline 91120 & $11120 \pm 428$ & $3.881 \pm 0.076$ & $295 \pm 21$ & $12592 \pm 244$ & $4.171 \pm 0.050$ & $310 \pm 16$ & $407 \pm 21$ & $70.7 \pm 2.7$ \\
\hline 91465 & $17389 \pm 415$ & $3.518 \pm 0.057$ & $266 \pm 16$ & $19338 \pm 582$ & $3.704 \pm 0.079$ & $285 \pm 20$ & $401 \pm 28$ & $67.3 \pm 3.7$ \\
\hline 105435 & $22360 \pm 589$ & $3.916 \pm 0.075$ & $260 \pm 17$ & \pm 446 & $3.942 \pm 0.053$ & $263 \pm 14$ & $527 \pm 29$ & $41.6 \pm 2.3$ \\
\hline 105521 & $16371 \pm 451$ & $3.097 \pm 0.060$ & $120 \pm 07$ & $17490 \pm 409$ & $3.124 \pm 0.052$ & $128 \pm 07$ & $320 \pm 19$ & $33.4 \pm 1.1$ \\
\hline 109387 & $13982 \pm 392$ & $3.479 \pm 0.061$ & $200 \pm 12$ & $15383 \pm 357$ & $3.582 \pm 0.054$ & $209 \pm 12$ & $362 \pm 21$ & $50.0 \pm 2.6$ \\
\hline 110432 & $20324 \pm 344$ & $3.638 \pm 0.042$ & $400 \pm 21$ & $24070 \pm 603$ & $3.950 \pm 0.074$ & $419 \pm 28$ & $485 \pm 32$ & $85.4 \pm 0.3$ \\
\hline 112078 & $16484 \pm 409$ & $4.028 \pm 0.063$ & $302 \pm 18$ & $18398 \pm 331$ & $4.281 \pm 0.051$ & $327 \pm 17$ & $506 \pm 27$ & $56.3 \pm 3.6$ \\
\hline 112091 & $20349 \pm 541$ & $3.934 \pm 0.073$ & $210 \pm 14$ & $21649 \pm 638$ & $3.944 \pm 0.084$ & $221 \pm 15$ & $492 \pm 35$ & $36.0 \pm 1.9$ \\
\hline 120324 & $22554 \pm 661$ & $3.906 \pm 0.085$ & $159 \pm 11$ & $22698 \pm 533$ & $3.904 \pm 0.067$ & $166 \pm 10$ & $508 \pm 32$ & $25.7 \pm 1.3$ \\
\hline 120991 & $22214 \pm 631$ & $3.685 \pm 0.079$ & $70 \pm 04$ & $22063 \pm 414$ & $3.679 \pm 0.049$ & $75 \pm 04$ & $429 \pm 23$ & $13.0 \pm 0.5$ \\
\hline 124367 & $17508 \pm 429$ & $3.757 \pm 0.061$ & $295 \pm 18$ & $19720 \pm 310$ & $3.914 \pm 0.041$ & \pm 16 & $446 \pm 22$ & $62.9 \pm 5.1$ \\
\hline 127972 & $19798 \pm 525$ & $3.846 \pm 0.071$ & $310 \pm 20$ & \pm 565 & $3.969 \pm 0.074$ & $326 \pm 21$ & $500 \pm 33$ & $57.8 \pm 3.8$ \\
\hline 131492 & $18353 \pm 576$ & $3.535 \pm 0.078$ & $185 \pm 13$ & $19660 \pm 392$ & $3.564 \pm 0.050$ & $195 \pm 11$ & $395 \pm 22$ & $41.0 \pm 1.9$ \\
\hline 135734 & $12705 \pm 240$ & $3.737 \pm 0.044$ & $278 \pm 14$ & $14483 \pm 383$ & $4.005 \pm 0.064$ & $282 \pm 17$ & $391 \pm 24$ & $68.7 \pm 4.0$ \\
\hline 137387 & $22920 \pm 456$ & $4.162 \pm$ & 250 & \pm 550 & $4.167 \pm 0.069$ & 16 & $573 \pm 36$ & $34.6 \pm 1.8$ \\
\hline 138749 & $14457 \pm 401$ & $3.745 \pm$ & $340 \pm 21$ & \pm 566 & $3.932 \pm 0.084$ & $345 \pm 24$ & $441 \pm 31$ & $79.7 \pm 3.8$ \\
\hline 142184 & $21246 \pm 397$ & $4.200 \pm 0.053$ & $340 \pm 18$ & $22649 \pm 640$ & $4.238 \pm 0.085$ & $342 \pm 24$ & $574 \pm 41$ & $51.5 \pm 2.4$ \\
\hline 142926 & $12076 \pm 403$ & $3.917 \pm 0.071$ & $336 \pm 22$ & $13866 \pm 339$ & $4.188 \pm 0.061$ & $338 \pm 20$ & $424 \pm 25$ & $73.8 \pm 2.0$ \\
\hline 142983 & $17645 \pm 554$ & $3.845 \pm$ & $390 \pm 27$ & $20104 \pm 389$ & $4.041 \pm 0.053$ & $407 \pm 22$ & $501 \pm 28$ & $67.4 \pm 3.7$ \\
\hline 148184 & $28783 \pm 727$ & $3.913 \pm$ & $144 \pm 10$ & 287 & $3.908 \pm 0.065$ & $151 \pm 09$ & $580 \pm 36$ & $20.0 \pm 0.9$ \\
\hline 149438 & $28959 \pm 526$ & $3.739 \pm$ & $15 \pm$ & 285 & $3.732 \pm 0.063$ & & $536 \pm 33$ & $2.3 \pm 0.1$ \\
\hline 149757 & $26378 \pm 729$ & $3.795 \pm 0.086$ & $340 \pm 25$ & $28797 \pm 638$ & $4.047 \pm 0.070$ & $352 \pm 22$ & $534 \pm 34$ & $60.7 \pm 3.3$ \\
\hline 157042 & $21787 \pm 533$ & $4.062 \pm 0.070$ & $340 \pm 22$ & $23711 \pm 639$ & $4.310 \pm 0.084$ & $348 \pm 24$ & $572 \pm 40$ & $53.1 \pm 3.7$ \\
\hline 158427 & $18044 \pm 310$ & $3.991 \pm$ & $290 \pm 15$ & \pm 327 & $4.232 \pm 0.047$ & $305 \pm 15$ & $477 \pm 24$ & $51.3 \pm 3.2$ \\
\hline 162732 & $13523 \pm 243$ & $3.754 \pm$ & $310 \pm 16$ & \pm 560 & $3.975 \pm 0.087$ & $321 \pm 23$ & $431 \pm 31$ & $72.9 \pm 2.6$ \\
\hline 164284 & $21609 \pm 523$ & $3.943 \pm 0.068$ & $280 \pm 17$ & $22822 \pm 670$ & $3.978 \pm 0.087$ & $287 \pm 21$ & $513 \pm 37$ & $48.0 \pm 2.4$ \\
\hline 167128 & $17396 \pm 608$ & $3.645 \pm 0.086$ & $48 \pm 03$ & $17254 \pm 575$ & $3.637 \pm 0.082$ & $55 \pm 03$ & $389 \pm 28$ & $10.6 \pm 0.3$ \\
\hline 173948 & $22075 \pm 472$ & $3.599 \pm$ & $140 \pm 08$ & $22212 \pm 517$ & $3.606 \pm 0.062$ & $150 \pm 09$ & $430 \pm 27$ & $27.3 \pm 1.1$ \\
\hline 174237 & $17683 \pm 556$ & $3.764 \pm$ & 16 & \pm 441 & $3.773 \pm 0.061$ & 10 & $425 \pm 26$ & $33.2 \pm 1.5$ \\
\hline 175869 & $12241 \pm 496$ & $3.254 \pm 0.077$ & $175 \pm 12$ & $13442 \pm 441$ & $3.474 \pm 0.069$ & $182 \pm 12$ & $311 \pm 20$ & $52.0 \pm 2.1$ \\
\hline 178175 & $18939 \pm 286$ & $3.489 \pm 0.035$ & $105 \pm 05$ & $18976 \pm 561$ & $3.493 \pm 0.074$ & $111 \pm 07$ & $396 \pm 27$ & $22.4 \pm 1.1$ \\
\hline 183656 & $12626 \pm 524$ & $3.295 \pm 0.081$ & $270 \pm 20$ & $14642 \pm 360$ & $3.551 \pm 0.056$ & $284 \pm 16$ & $354 \pm 21$ & $76.7 \pm 4.9$ \\
\hline 183914 & $13218 \pm 445$ & $3.830 \pm 0.074$ & $220 \pm 14$ & $14422 \pm 278$ & $3.867 \pm 0.048$ & $228 \pm 12$ & $387 \pm 21$ & $48.7 \pm 2.3$ \\
\hline 184915 & $27076 \pm 540$ & $3.492 \pm 0.054$ & $229 \pm 13$ & $28888 \pm 606$ & $3.528 \pm 0.060$ & $244 \pm 15$ & $479 \pm 29$ & $41.1 \pm 2.5$ \\
\hline 185037 & $12058 \pm 212$ & $3.768 \pm 0.042$ & $280 \pm 14$ & $13586 \pm 523$ & $4.002 \pm 0.086$ & $287 \pm 20$ & $410 \pm 29$ & $68.4 \pm 3.6$ \\
\hline 187811 & $18086 \pm 583$ & $3.810 \pm 0.083$ & $245 \pm 17$ & $19682 \pm 336$ & $3.849 \pm 0.044$ & $258 \pm 13$ & $434 \pm 22$ & $48.9 \pm 2.4$ \\
\hline 189687 & $18106 \pm 379$ & $3.455 \pm 0.050$ & $200 \pm 11$ & $19336 \pm 319$ & $3.498 \pm 0.039$ & $212 \pm 11$ & $395 \pm 20$ & $46.6 \pm 2.3$ \\
\hline 191610 & $18353 \pm 516$ & $3.718 \pm 0.072$ & $300 \pm 20$ & $20671 \pm 587$ & $3.983 \pm 0.079$ & $318 \pm 22$ & $442 \pm 30$ & $63.7 \pm 3.9$ \\
\hline 192044 & $12570 \pm 490$ & $3.366 \pm 0.077$ & $245 \pm 17$ & $14452 \pm 221$ & $3.573 \pm 0.036$ & $248 \pm 12$ & $332 \pm 16$ & $71.1 \pm 3.1$ \\
\hline 193911 & $12467 \pm 424$ & $3.090 \pm 0.065$ & $160 \pm 10$ & $13434 \pm 352$ & $3.194 \pm 0.053$ & $168 \pm 10$ & $292 \pm 17$ & $49.1 \pm 2.0$ \\
\hline 194335 & $22120 \pm 373$ & $4.071 \pm 0.047$ & $360 \pm 19$ & $24357 \pm 458$ & $4.185 \pm 0.055$ & $363 \pm 20$ & $581 \pm 32$ & $57.6 \pm 3.9$ \\
\hline 198183 & $13925 \pm 332$ & $3.167 \pm 0.049$ & $125 \pm 07$ & $14233 \pm 404$ & $3.195 \pm 0.059$ & $133 \pm 08$ & $300 \pm 19$ & $35.6 \pm 1.5$ \\
\hline 200120 & $21750 \pm 655$ & $3.784 \pm 0.085$ & $379 \pm 27$ & $24808 \pm 520$ & $3.966 \pm 0.061$ & $387 \pm 23$ & $530 \pm 32$ & $73.2 \pm 3.2$ \\
\hline 201733 & $16350 \pm 504$ & $3.943 \pm 0.077$ & $340 \pm 23$ & $18410 \pm 375$ & $4.176 \pm 0.056$ & $357 \pm 20$ & $481 \pm 27$ & $68.4 \pm 4.8$ \\
\hline 203374 & $24645 \pm 660$ & $3.458 \pm 0.076$ & $333 \pm 23$ & $27662 \pm 651$ & $3.663 \pm 0.070$ & $342 \pm 22$ & $477 \pm 31$ & $70.7 \pm 3.7$ \\
\hline 203467 & $17087 \pm 521$ & $3.377 \pm 0.072$ & $153 \pm 10$ & $18249 \pm 575$ & $3.404 \pm 0.077$ & $165 \pm 11$ & $356 \pm 25$ & $38.2 \pm 1.4$ \\
\hline 205637 & $17801 \pm 470$ & $3.442 \pm 0.063$ & $225 \pm 14$ & $19348 \pm 351$ & $3.673 \pm 0.045$ & $238 \pm 12$ & $395 \pm 21$ & $54.7 \pm 2.7$ \\
\hline
\end{tabular}


Table 9. continued.

\begin{tabular}{cccc|ccccc}
\hline \hline \multicolumn{3}{c|}{ Apparent parameters } & \multicolumn{5}{c}{ Mode of the pnrc parameters } \\
$\mathrm{HD}$ & $T_{\mathrm{eff}} \pm \epsilon$ & $\log g \pm \epsilon$ & $V \sin i \pm \epsilon$ & $T_{\mathrm{eff}} \pm 1 \sigma$ & $\log g \pm 1 \sigma$ & $V \sin i \pm 1 \sigma$ & $V_{\mathrm{c}} \pm 1 \sigma$ & $i^{o} \pm 1 \sigma$ \\
\hline 206773 & $29154 \pm 746$ & $3.885 \pm 0.083$ & $390 \pm 28$ & $31800 \pm 547$ & $4.143 \pm 0.052$ & $399 \pm 21$ & $617 \pm 33$ & $62.1 \pm 3.6$ \\
208057 & $17974 \pm 396$ & $3.675 \pm 0.055$ & $100 \pm 05$ & $17977 \pm 510$ & $3.672 \pm 0.071$ & $110 \pm 07$ & $391 \pm 26$ & $20.6 \pm 0.9$ \\
208682 & $22114 \pm 652$ & $3.977 \pm 0.086$ & $307 \pm 22$ & $23814 \pm 617$ & $4.019 \pm 0.077$ & $314 \pm 21$ & $525 \pm 36$ & $53.3 \pm 2.9$ \\
209014 & $12016 \pm 408$ & $3.155 \pm 0.064$ & $320 \pm 21$ & $14694 \pm 509$ & $3.571 \pm 0.077$ & $357 \pm 21$ & $343 \pm 24$ & $90.0 \pm 1.0$ \\
209409 & $12942 \pm 402$ & $3.701 \pm 0.067$ & $280 \pm 18$ & $14562 \pm 514$ & $3.978 \pm 0.082$ & $282 \pm 20$ & $391 \pm 27$ & $69.9 \pm 3.4$ \\
209522 & $16581 \pm 408$ & $3.704 \pm 0.060$ & $275 \pm 16$ & $18507 \pm 279$ & $3.886 \pm 0.039$ & $299 \pm 14$ & $430 \pm 21$ & $66.6 \pm 4.3$ \\
210129 & $13515 \pm 240$ & $3.421 \pm 0.040$ & $130 \pm 06$ & $14127 \pm 444$ & $3.439 \pm 0.068$ & $140 \pm 09$ & $326 \pm 21$ & $33.8 \pm 1.4$ \\
212076 & $19270 \pm 326$ & $3.726 \pm 0.043$ & $98 \pm 05$ & $19236 \pm 419$ & $3.721 \pm 0.056$ & $103 \pm 06$ & $439 \pm 26$ & $18.6 \pm 0.9$ \\
212571 & $26061 \pm 736$ & $3.915 \pm 0.088$ & $230 \pm 17$ & $27574 \pm 645$ & $3.926 \pm 0.072$ & $233 \pm 15$ & $558 \pm 37$ & $33.6 \pm 1.7$ \\
214168 & $25827 \pm 434$ & $4.200 \pm 0.049$ & $300 \pm 16$ & $27647 \pm 688$ & $4.211 \pm 0.081$ & $305 \pm 21$ & $619 \pm 43$ & $40.0 \pm 1.7$ \\
214748 & $11966 \pm 356$ & $3.375 \pm 0.059$ & $200 \pm 12$ & $13294 \pm 437$ & $3.606 \pm 0.070$ & $205 \pm 13$ & $317 \pm 21$ & $56.9 \pm 2.6$ \\
217050 & $17893 \pm 509$ & $3.571 \pm 0.070$ & $340 \pm 22$ & $20723 \pm 612$ & $3.871 \pm 0.082$ & $355 \pm 25$ & $443 \pm 31$ & $78.5 \pm 2.1$ \\
217543 & $17237 \pm 584$ & $3.685 \pm 0.083$ & $325 \pm 24$ & $19569 \pm 366$ & $3.933 \pm 0.050$ & $335 \pm 18$ & $440 \pm 24$ & $74.9 \pm 3.9$ \\
217675 & $14052 \pm 439$ & $3.229 \pm 0.065$ & $274 \pm 19$ & $16741 \pm 469$ & $3.460 \pm 0.066$ & $289 \pm 17$ & $343 \pm 22$ & $90.0 \pm 1.0$ \\
217891 & $14359 \pm 295$ & $3.672 \pm 0.048$ & $95 \pm 05$ & $14376 \pm 449$ & $3.668 \pm 0.070$ & $100 \pm 06$ & $367 \pm 24$ & $20.7 \pm 1.0$ \\
224544 & $14799 \pm 279$ & $3.364 \pm 0.042$ & $260 \pm 14$ & $16557 \pm 460$ & $3.560 \pm 0.066$ & $270 \pm 17$ & $373 \pm 24$ & $72.8 \pm 2.6$ \\
224559 & $15914 \pm 329$ & $3.597 \pm 0.049$ & $300 \pm 16$ & $18338 \pm 412$ & $3.809 \pm 0.058$ & $312 \pm 18$ & $427 \pm 25$ & $75.8 \pm 3.7$ \\
224686 & $11286 \pm 218$ & $3.671 \pm 0.044$ & $290 \pm 16$ & $12800 \pm 281$ & $3.948 \pm 0.052$ & $300 \pm 16$ & $380 \pm 21$ & $76.1 \pm 2.7$ \\
\hline
\end{tabular}

